UWO-TH-07/10

WIS/07/07-JUNE-DPP

SLAC-PUB-12552

hep-th/0706.1768

\title{
The black hole in the throat - thermodynamics of strongly coupled cascading gauge theories
}

\author{
Ofer Aharony ${ }^{1,2}$, Alex Buchel ${ }^{3,4}$ and Patrick Kerner ${ }^{3}$ \\ ${ }^{1}$ Department of Particle Physics, Weizmann Institute of Science, \\ Rehovot 76100, Israel \\ ${ }^{2}$ SITP, Department of Physics and SLAC, Stanford University, \\ Stanford, California 94305, USA \\ ${ }^{3}$ Department of Applied Mathematics \\ University of Western Ontario \\ London, Ontario N6A 5B7, Canada \\ ${ }^{4}$ Perimeter Institute for Theoretical Physics \\ Waterloo, Ontario N2J 2W9, Canada
}

\begin{abstract}
We numerically construct black hole solutions corresponding to the deconfined, chirally symmetric phase of strongly coupled cascading gauge theories at various temperatures. We compute the free energy as a function of the temperature, and we show that it becomes positive below some critical temperature, indicating the possibility of a first order phase transition at which the theory deconfines and restores the chiral symmetry.
\end{abstract}




\section{Introduction and Summary}

One of the most interesting outcomes of the AdS/CFT correspondence $[1,2,3,4]$ is the ability to study quantitatively the deconfined phase of $3+1$ dimensional gauge theories, something which cannot be done analytically for QCD (except at temperatures much higher than the QCD scale). For strongly coupled large $N$ gauge theories which have (at zero temperature) a dual description given by a weakly curved string background, the deconfined phase has a dual description in terms of a black hole (black brane) background which can be reliably studied in the supergravity approximation. Of course, theories with a weakly curved dual are rather different from QCD in various ways, but one can still hope that their deconfined phase will not behave that differently from that of QCD, and in some cases these theories are even continuously connected to (large $N)$ QCD by varying a dimensionless parameter, and one could hope that the dependence on this parameter is small (at least at temperatures of order the deconfinement temperature).

The simplest theory to study in this way, on which most of the research thus far has focused, is the strong coupling limit of $\mathcal{N}=4$ supersymmetric Yang-Mills theory, whose deconfined phase has a very simple description as a black hole in anti-de Sitter space [5]. This theory does not confine at low temperatures, but its deconfined phase still seems to exhibit many similarities to that of QCD. Obviously, it would be nice to have additional examples of $3+1$ dimensional deconfined theories which can be studied quantitatively by using their gravity dual, and, in particular, examples of deconfined phases of confining theories, in which one could study the dependence on the temperature compared to the deconfinement scale ${ }^{1}$. So far there is only one known example of such a deconfined background, which is that corresponding to $4+1$ dimensional supersymmetric Yang-Mills theory (with a specific UV completion) compactified on a circle with anti-periodic boundary conditions for fermions [5, 6]. It would be nice to have additional examples, especially since in the example above the physics at the deconfinement scale is really five dimensional rather than four dimensional.

In this paper we study the deconfined phase of the confining "cascading gauge theories" constructed by Klebanov and collaborators [7, 8, 9]. The equations determining

\footnotetext{
${ }^{1}$ Note that in any large $N$ gauge theory with a weakly curved string theory dual the deconfinement transition is a first order phase transition. This is similar to large $N$ QCD, but it is different from QCD itself, so one should be careful when comparing the behavior of such theories near the phase transition to that of QCD.
} 
the corresponding black hole solutions are quite complicated, and have no known analytic solutions. At very high temperatures it is possible to find analytic solutions in an expansion in inverse powers of the logarithm of the temperature, and the leading order solution in this expansion was found in [10]. This solution shows that at high temperatures the number of degrees of freedom in the theory grows as the square of the logarithm of the temperature $[11,12,10]$. In this paper we numerically solve the equations for a wide range of values of the temperature, in the supergravity approximation, and use the solutions to analyze the thermodynamics of the deconfined phase ${ }^{2}$.

At low temperatures the "cascading gauge theories" spontaneously break a discrete chiral symmetry (and also a continuous $U(1)_{B}$ symmetry) $[9,14,15,16]$. At high temperatures one expects these symmetries to be restored, but apriori it is not obvious whether there is a single phase transition from a low-temperature phase with confinement and chiral symmetry breaking to a high-temperature phase with no confinement and chiral symmetry restored, or whether additional phases also exist. In this paper we only study deconfined phases in which the chiral symmetry is restored; we plan to study the possibility of having other phases in the future [17]. In the classical supergravity approximation the free energy of the low temperature confined phase vanishes (since it only arises at one-loop), and thus the phase transition to a black hole background occurs at the lowest temperature for which the free energy of a black hole background starts becoming negative. Assuming that this transition goes directly to the chirally symmetric black holes that we construct, we find that there is a first order deconfinement transition at a temperature $T_{\text {critical }}=0.614111(3) \Lambda$, according to a specific definition of the strong coupling scale $\Lambda$ that we describe in section $5^{3}$. The black hole backgrounds continue to exist also at lower temperatures, but they have positive free energies so they no longer dominate the thermodynamics. Presumably, as the temperature is lowered further, the black hole backgrounds eventually become singular; in this paper we only compute the numerical solutions until a temperature

\footnotetext{
${ }^{2}$ Similar numerical solutions were studied in [13], but we do not understand the parametrization used there to analyze the solutions. Presumably, our solutions should be identical to (some of) the solutions of [13], but our parametrization allows for a direct computation of the thermodynamical properties of the solutions.

${ }^{3}$ It is easy to translate this definition to other definitions of the strong coupling scale, such as the mass gap. Note that, as in all theories with a gravity approximation, the square root of the confining string tension is not a useful measure of the strong coupling scale, since it must be much larger than all other measures of this scale for gravity to be a good approximation.
} 
slightly below the deconfinement temperature, so we do not see this.

There are several interesting directions for further study. We are currently working on checking whether the solutions we find are stable to deformations which break the chiral symmetry, in order to see if there are signs of a deconfined non-chirallysymmetric phase appearing at intermediate temperatures [17]. The black hole solutions that we find (numerically) can be used for a detailed analysis of the properties of the deconfined phase (for instance its hydrodynamical properties [18] or jet quenching $[19,20])$; it would be interesting to understand the similarities and differences between these properties and those of deconfined conformal theories. It is possible to add flavors in various ways to the "cascading gauge theories" [21, 22, 23, 24, 25, 26, 27], and it would be interesting to study the flavor physics in the deconfined phase, and whether there are any phase transitions associated with the flavor sector.

Our study is purely in the supergravity approximation; it would be interesting to study the corrections to this approximation, in particular those coming from string theory corrections to the supergravity action. The cascading gauge theories have a continuous dimensionless parameter such that in one extreme of this parameter supergravity is a good approximation, while in the other extreme they reduce to a standard $\mathcal{N}=1$ supersymmetric Yang-Mills (SYM) theory. At zero temperature supersymmetry tells us that the dependence on this parameter is smooth. However, it is not obvious if the behavior of the deconfined phase is smooth as this parameter in changed; in fact, it seems plausible [28] that as in other similar cases [6] there would be a phase transition in this phase, since the geometry in the supergravity regime does not have any cycles shrinking at the horizon (except for the thermal $S^{1}$ ), while in the SYM regime one expects the transition to be independent of the KK modes so a two-cycle should still shrink (as it does in the confined phase). Of course, even in the absence of such a phase transition, the behavior of the theory could be modified as the dimensionless parameter is changed, so our analysis does not teach us directly about the phase structure of the large $N \mathcal{N}=1$ SYM theory.

This paper is organized as follows. In section 2 we describe our ansatz for the black hole solutions and the equations of motion that it leads to. In section 3 we describe the boundary conditions for these equations. In section 4 we analyze the meaning of the parameters appearing in these boundary conditions, and show how to translate them into physical quantities such as the temperature and free energy. In section 5 we describe our numerical procedure and present the "bare" numerical results. In section 
6 we translate these results into physical properties, and present the results for physical quantities such as the free energy and the expectation values of various operators as a function of the temperature. An appendix contains a perturbative analysis of the very high temperature solutions; this is useful both in order to make sure that our analysis is valid by verifying that it is consistent (at least at very high temperatures) with the first law of thermodynamics, and in order to test our numerical solutions at very high temperatures by testing their agreement with the perturbative expansion.

\section{The equations for the cascading black hole}

In this paper we compute the metrics corresponding to the finite temperature behavior of the "cascading gauge theory" found in $[7,8,9]^{4}$, which may be thought of as a specific $S U(K) \times S U(K+M) \mathcal{N}=1$ supersymmetric gauge theory, with a number of colors $K$ which runs logarithmically with the energy scale $[9,11,12,30,31]$. The "cascading gauge theory" has a single dimensionless parameter (in addition to the integer $M$ ), which in the gravitational description of this theory can be taken to be $g_{s} M$ where $g_{s}$ is the string coupling (which is constant in the zero temperature solution) and $M$ is the RR 3-form flux (corresponding to the number of fractional branes). When this parameter is large, the gravitational description of the background is valid at all scales. On the other hand, when it is small, the theory at low energies reduces exactly to the $\mathcal{N}=1$ SYM theory, but the gravity dual is highly curved. We will only analyze the theory in the regime of large $g_{s} M$, where the gravitational approximation is good and all radii of curvature are large compared to the string scale.

As in any other confining background, the low temperature behavior of this theory is governed by a gas of hadrons; the gravity dual of this description is simply given by a thermal identification $(t \equiv t+1 / T)$ of the zero temperature solution found in [9]. As the temperature is increased one expects the theory to deconfine; in the gravitational dual, deconfined phases are described by black holes (whose horizon fills all of space, so they are really black branes). Our goal in this paper will be to compute the gravitational backgrounds corresponding to the deconfined phase of the cascading gauge theory. Note that the low temperature phase is stable all the way up to the Hagedorn temperature of the confining theory (related to the confining string tension); when the gravitational approximation is valid, this temperature is very large compared to the characteristic

\footnotetext{
${ }^{4}$ See [29] for a recent review of this theory.
} 
mass scale of the gravitational background (which determines the mass of the low-lying hadrons). Thus, as in all other cases of confining backgrounds with gravity duals, we expect the deconfinement transition to occur at a temperature which is much smaller than the Hagedorn temperature, which means that it should be a first order phase transition.

The cascading gauge theory has a $Z_{2 M}$ chiral symmetry $[9,15]$ which is spontaneously broken to $Z_{2}$ at low temperatures (by gaugino condensation in the limit where the theory is a pure SYM theory), and it has a $U(1)_{B}$ symmetry which is also spontaneously broken $[14,16]$. At high enough temperatures we expect these symmetries to be restored [11,32]; this expectation is confirmed by the analysis of the asymptotically high temperature black hole solutions in [10]. Apriori it is not obvious if the deconfinement transition happens together with the global symmetry restoration transitions, or if the transitions are separate. In this paper we will only look for solutions which preserve the chiral symmetry and the $U(1)_{B}$ symmetry; the stability of these solutions with respect to chiral-symmetry-breaking deformations will be analyzed in [17]. We also assume that the solutions preserve the $S U(2) \times S U(2)$ global symmetry of the theory, which is preserved also at low temperatures so it is reasonable to assume that it is preserved at all temperatures.

The form of the gravitational background of the cascading gauge theory at large radial variables (close to the boundary) was found in [8] and is known as the KlebanovTseytlin solution; this form preserves the full global symmetry. The solution at any temperature is expected to asymptote to this background near the boundary (the "UV region"). In the zero temperature solution [9] the $Z_{2 M}$ and $U(1)_{B}$ symmetries are broken far from the boundary (in the "IR region"), but we will look for solutions where they are preserved. Recall that the solution for $M=0$ (where the theory does not cascade) is [33] $A d S_{5} \times T^{1,1}$, and that the solution of [8] has a similar form but with the radii of curvature (and the flux) varying logarithmically in the radial coordinate. Since we are looking for solutions that preserve the full global symmetry, we can perform a Kaluza-Klein reduction on the $T^{1,1}$, and leave only the fields which are singlets of the global symmetry group. In general there are 5 such fields ${ }^{5}$; the five dimensional graviton and 4 scalar fields. In the $M=0$ limit the scalar fields have scaling dimensions $\Delta=4,4,6,8$. The scalar fields are various linear combinations of

\footnotetext{
${ }^{5}$ We only consider the fields which are turned on in the solutions that we are interested in; other fields, such as the type IIB axion, are consistently set to zero.
} 
the dilaton, the overall volume of the $T^{1,1}$, the relative size of the circle in $T^{1,1}$ (thinking of $T^{1,1}$ as a circle fibration over two 2-spheres), and one mode coming from the RR fields.

We are looking for black hole solutions that preserve spatial rotational and translational invariance, as well as time translation invariance, so we can always choose a form of the five dimensional metric where only 3 components are not vanishing $-G_{00}$, $G_{i i}(i=1,2,3)$ and $G_{r r}$ (where $r$ is the radial position). We can use the freedom of reparametrizing the radial coordinate to eliminate one of these degrees of freedom we will choose our radial coordinate $x$ to be defined by

$$
\frac{G_{00}}{G_{i i}}=-(1-x)^{2}
$$

(with no summation over $i$ ). This choice is convenient since at the boundary we expect the metric to be Lorentz-invariant so $x \rightarrow 0$, while at the horizon $G_{00}$ vanishes so $x \rightarrow 1$; the range of the radial coordinate in our parametrization is thus $x \in(0,1)$. Note that in the conformal $M=0$ case there is a simple form of the black hole solution in this parametrization, which is given by

$$
d s_{10}^{2}=\xi^{2}\left(2 x-x^{2}\right)^{-1 / 2}\left(-(1-x)^{2} d t^{2}+d x_{1}^{2}+d x_{2}^{2}+d x_{3}^{2}\right)+\frac{d x^{2}}{4\left(2 x-x^{2}\right)^{2}}+\left(d T^{1,1}\right)^{2},
$$

where $\left(d T^{1,1}\right)^{2}$ is the metric on $T^{1,1}$, and the constant $\xi$ is related to the temperature $T$ as follows:

$$
\xi=\pi T
$$

Motivated by the form of (2.2), we write down the most general ansatz for a black hole metric preserving all the symmetries $\mathrm{as}^{6}$ :

$$
\begin{aligned}
d s_{10}^{2}= & h^{-1 / 2}\left(2 x-x^{2}\right)^{-1 / 2}\left(-(1-x)^{2} d t^{2}+d x_{1}^{2}+d x_{2}^{2}+d x_{3}^{2}\right)+G_{x x}(d x)^{2} \\
& +h^{1 / 2}\left[f_{2}\left(e_{\psi}^{2}\right)+f_{3} \sum_{a=1}^{2}\left(e_{\theta_{a}}^{2}+e_{\phi_{a}}^{2}\right)\right]
\end{aligned}
$$

where $h, f_{2}$ and $f_{3}$ are some functions of the radial coordinate $x$. There is also a dilaton $g(x)$, and form fields given by

$$
\begin{aligned}
& F_{3}=P e_{\psi} \wedge\left(e_{\theta_{1}} \wedge e_{\phi_{1}}-e_{\theta_{2}} \wedge e_{\phi_{2}}\right), \quad B_{2}=\frac{K}{2 P}\left(e_{\theta_{1}} \wedge e_{\phi_{1}}-e_{\theta_{2}} \wedge e_{\phi_{2}}\right), \\
& F_{5}=\mathcal{F}_{5}+\star \mathcal{F}_{5}, \quad \mathcal{F}_{5}=-K e_{\psi} \wedge e_{\theta_{1}} \wedge e_{\phi_{1}} \wedge e_{\theta_{2}} \wedge e_{\phi_{2}}
\end{aligned}
$$

\footnotetext{
${ }^{6}$ The frames $\left\{e_{\theta_{a}}, e_{\phi_{a}}\right\}$ are defined as in [30], such that the metric on a unit size $T^{1,1}$ is given by $\left(e_{\psi}^{2}\right)+\sum_{a=1}^{2}\left(e_{\theta_{a}}^{2}+e_{\phi_{a}}^{2}\right)$.
} 
where $K$ is a function of the radial coordinate $x$. The constant $P$ appearing in (2.5) is a constant times the quantized flux $M$; we will write down the precise constant in terms of the five dimensional Newton's constant below. We will find it simpler to work in terms of $P$ rather than $M$, and we will only go back to using the integer flux $M$ in the final section. After we gauge-fixed the radial coordinate by (2.1), we have a constraint equation coming from the equation of motion of this variable; we can use this equation to solve for $G_{x x}$, which is given by

$$
\begin{aligned}
G_{x x}= & \frac{\sqrt{h} f_{3}^{2}}{2(x-1) P^{2} g^{2}(2-x)^{2} x^{2} \Delta}\left(12 P^{2} f_{3}^{2} g^{2} f_{2} h^{2}(1-x)\right. \\
& +f_{2} x^{2}\left(2 P^{2} f_{3}^{2} g^{2} h^{\prime 2}-12 P^{2} g^{2} h^{2} f_{3}^{\prime 2}+K^{\prime 2} h g+2 P^{2} h^{2} f_{3}^{2} g^{\prime 2}\right)(x-1)(2-x)^{2} \\
& -4 x P^{2} f_{3} g^{2} h f_{2}(2-x)\left(x^{2}-2 x+2\right)\left(h^{\prime} f_{3}+4 f_{3}^{\prime} h\right) \\
& \left.+4 x P^{2} f_{3}(2-x) g^{2} h^{2}\left(2 x f_{3}^{\prime}(1-x)(2-x)-\left(x^{2}-2 x+2\right) f_{3}\right) f_{2}^{\prime}\right),
\end{aligned}
$$

with

$$
\Delta \equiv K^{2}+8 h^{2} f_{3}^{2} f_{2}\left(f_{2}-6 f_{3}\right)+2 h f_{3}^{2} P^{2} g .
$$

All in all, we have 5 scalar functions of $x$ that we need to solve for : $h, f_{2}, f_{3}, g$ and $K$. We can derive the equations of motion for these fields, in the supergravity approximation, either directly from the ten dimensional type IIB supergravity action, or by first reducing this action to five dimensions and then deriving the equations of motion. The equations that we find take the following rather complicated form :

$$
\begin{aligned}
0= & h^{\prime \prime}-\left[8 h f_{2}\left(f_{2}-6 f_{3}\right)+g P^{2}\right] \frac{f_{3}^{2} h^{\prime 2}}{\Delta}+\left[8 x h^{2} f_{3}^{2} f_{2}\left(f_{2}-6 f_{3}\right)(x-2)\right. \\
& \left.+K^{2}\left(3 x^{2}-6 x+4\right)+4 h f_{3}^{2} P^{2} g(1-x)^{2}\right] \frac{h^{\prime}}{x(1-x)(2-x) \Delta} \\
& -6\left(K^{2}+h f_{3}^{2} P^{2} g\right) \frac{h f_{3}^{\prime 2}}{f_{3}^{2} \Delta}+\left[8 h^{2} f_{3}^{2} f_{2}\left(f_{2}-6 f_{3}\right)+3 K^{2}+4 h f_{3}^{2} P^{2} g\right] \frac{K^{\prime 2}}{4 g f_{3}^{2} P^{2} \Delta} \\
& +\left(K^{2}+h f_{3}^{2} P^{2} g\right) \frac{h g^{\prime 2}}{g^{2} \Delta}-2 h\left(K^{2}+h f_{3}^{2} P^{2} g\right)\left[2 x f_{3}^{\prime}(1-x)(2-x)-\left(x^{2}-2 x+2\right) f_{3}\right] \\
& \times \frac{f_{2}^{\prime}}{f_{3} f_{2} x(1-x)(2-x) \Delta}+8\left(x^{2}-2 x+2\right)\left(K^{2}+h f_{3}^{2} P^{2} g\right) \frac{h f_{3}^{\prime}}{x(1-x)(2-x) f_{3} \Delta} \\
& -2\left[7 h f_{3}^{2} P^{2} g+16 h^{2} f_{3}^{2} f_{2}\left(f_{2}-6 f_{3}\right)+5 K^{2}\right] \frac{h}{(2-x)^{2} x^{2} \Delta},
\end{aligned}
$$




$$
\begin{aligned}
& 0=f_{2}^{\prime \prime}-\frac{f_{2}^{\prime 2}}{f_{2}}-\left[4 x f_{3}^{\prime} f_{3} h(1-x)(2-x)\left(g P^{2}-8 f_{2}^{2} h\right)+8 f_{2} h^{2} f_{3}^{2}\left\{6 x f_{3}(x-2)\right.\right. \\
& \left.\left.+f_{2}\left(4-2 x+x^{2}\right)\right\}+x K^{2}(2-x)-4 h f_{3}^{2} P^{2} g(1-x)^{2}\right] \frac{f_{2}^{\prime}}{(1-x)(2-x) x \Delta} \\
& +f_{3}^{2}\left(g P^{2}-8 f_{2}^{2} h\right) \frac{f_{2} h^{\prime 2}}{h \Delta}-6 h\left(g P^{2}-8 f_{2}^{2} h\right) \frac{f_{2} f_{3}^{\prime 2}}{\Delta} \\
& -\left(K^{2}+24 f_{3}^{2} h^{2} f_{2}\left(f_{2}-2 f_{3}\right)\right) \frac{f_{2} K^{\prime 2}}{4 h f_{3}^{2} P^{2} g \Delta}+h f_{3}^{2}\left(g P^{2}-8 f_{2}^{2} h\right) \frac{f_{2} g^{\prime 2}}{g^{2} \Delta} \\
& +2 f_{3}^{2}\left(x^{2}-2 x+2\right)\left(g P^{2}-8 f_{2}^{2} h\right) \frac{f_{2} h^{\prime}}{(2-x)(1-x) x \Delta} \\
& +8 h f_{3}\left(x^{2}-2 x+2\right)\left(g P^{2}-8 f_{2}^{2} h\right) \frac{f_{2} f_{3}^{\prime}}{(2-x)(1-x) x \Delta} \\
& +2\left[16 f_{3}^{2} h^{2} f_{2}\left(2 f_{2}-3 f_{3}\right)+K^{2}-h f_{3}^{2} P^{2} g\right] \frac{f_{2}}{(x-2)^{2} x^{2} \Delta}, \\
& 0=f_{3}^{\prime \prime}-\left[2 P^{2} g h f_{3}^{2}+8 f_{3}^{2} f_{2} h^{2}\left(4 f_{2}-15 f_{3}\right)+K^{2}\right] \frac{f_{3}^{\prime 2}}{f_{3} \Delta}+4\left(f_{2}-3 f_{3}\right) \frac{f_{3}^{3} f_{2} h^{\prime 2}}{\Delta} \\
& +2\left(f_{2}-3 f_{3}\right) \frac{f_{3} h f_{2} K^{\prime 2}}{P^{2} g \Delta}+4\left(f_{2}-3 f_{3}\right) \frac{f_{3}^{3} h^{2} f_{2} g^{\prime 2}}{g^{2} \Delta} \\
& +8\left(x^{2}-2 x+2\right)\left(f_{2}-3 f_{3}\right) \frac{h f_{3}^{3} f_{2}}{(2-x)(1-x) x \Delta} h^{\prime} \\
& -8\left(f_{2}-3 f_{3}\right)\left[2 x f_{3}^{\prime}(1-x)(2-x)-\left(x^{2}-2 x+2\right) f_{3}\right] \frac{h^{2} f_{3}^{2} f_{2}^{\prime}}{(2-x)(1-x) x \Delta} \\
& +\left\{8 f_{2} f_{3}^{2}\left[\left(5 x^{2}-10 x+8\right) f_{2}-6 f_{3}\left(3 x^{2}-6 x+4\right)\right] h^{2}+2 x P^{2} g f_{3}^{2}(x-2) h\right. \\
& \left.+x K^{2}(x-2)\right\} \frac{f_{3}^{\prime}}{(1-x) x(2-x) \Delta}+\left[4 P^{2} g h f_{3}^{2}+2 K^{2}-8 f_{3}^{2} f_{2}\left(3 f_{3}+f_{2}\right) h^{2}\right] \\
& \times \frac{f_{3}}{x^{2}(2-x)^{2} \Delta} \\
& 0=K^{\prime \prime}-\frac{K K^{\prime 2}}{\Delta}+\left[h g f_{3}+2 h g f_{3}^{\prime}(1-x)+h f_{3} g^{\prime}(1-x)+g f_{3} h^{\prime}(1-x)\right] \frac{K^{\prime}}{g h f_{3}(x-1)} \\
& +12 P^{2} \frac{g K h f_{3}^{\prime 2}}{\Delta}-2 \frac{g P^{2} K f_{3}^{2} h^{\prime 2}}{h \Delta}-2 P^{2} \frac{K h f_{3}^{2} g^{\prime 2}}{g \Delta}+4\left[2 x f_{3}^{\prime}(1-x)(2-x)\right. \\
& \left.-\left(x^{2}-2 x+2\right) f_{3}\right] \frac{K g f_{3} P^{2} h f_{2}^{\prime}}{f_{2}(1-x)(2-x) x \Delta}-4 P^{2} g K\left(x^{2}-2 x+2\right) \frac{f_{3}^{2} h^{\prime}}{(2-x)(1-x) x \Delta} \\
& -16\left(x^{2}-2 x+2\right) \frac{K f_{3} P^{2} g h f_{3}^{\prime}}{(2-x)(1-x) x \Delta}+12 \frac{f_{3}^{2} h K P^{2} g}{x^{2}(2-x)^{2} \Delta},
\end{aligned}
$$




$$
\begin{aligned}
0= & g^{\prime \prime}-\frac{g^{\prime}}{1-x}-\left[8 h^{2} f_{3}^{2} f_{2}\left(f_{2}-6 f_{3}\right)+3 P^{2} g h f_{3}^{2}+K^{2}\right] \frac{g^{\prime 2}}{g \Delta}-P^{2} \frac{f_{3}^{2} g^{2} h^{\prime 2}}{h \Delta}+6 P^{2} \frac{h g^{2} f_{3}^{\prime 2}}{\Delta} \\
& +\left(8 h^{2} f_{3}^{2} f_{2}\left(f_{2}-6 f_{3}\right)+K^{2}\right) \frac{K^{\prime 2}}{4 P^{2} f_{3}^{2} h \Delta}-2 f_{3}^{2}\left(x^{2}-2 x+2\right) P^{2} \frac{g^{2} h^{\prime}}{(2-x)(1-x) x \Delta} \\
& +2 f_{3} g^{2} P^{2}\left[2 x f_{3}^{\prime}(1-x)(2-x)-\left(x^{2}-2 x+2\right) f_{3}\right] \frac{h f_{2}^{\prime}}{f_{2}(2-x)(1-x) x \Delta} \\
& -8\left(x^{2}-2 x+2\right) P^{2} \frac{g^{2} h f_{3} f_{3}^{\prime}}{(2-x)(1-x) x \Delta}+6 P^{2} \frac{g^{2} f_{3}^{2} h}{x^{2}(2-x)^{2} \Delta} .
\end{aligned}
$$

\section{Boundary conditions}

In order to solve the equations of motion (2.8)-(2.12) we need to specify boundary conditions, both at the asymptotic boundary and at the horizon. We will require that asymptotically the solution should match onto the Klebanov-Tseytlin (KT) solution, and that it should be regular near the horizon.

\subsection{The UV boundary conditions}

Near the boundary $x \rightarrow 0$ it is possible to solve the equations by a power series in $x$ and $\ln (x)$, whose leading term gives the KT solution. This expansion takes the general form :

$$
\begin{aligned}
h & =h_{0,0}-\frac{P^{2} g_{0}}{8 a_{0}^{2}} \ln (x)+\sum_{n=1}^{\infty} \sum_{k=1}^{n} h_{n, k} x^{n / 2} \ln ^{k}(x), \\
f_{2} & =a_{0}+\sum_{n=1}^{\infty} \sum_{k=1}^{n} a_{n, k} x^{n / 2} \ln ^{k}(x), \\
f_{3} & =a_{0}+\sum_{n=1}^{\infty} \sum_{k=1}^{n} b_{n, k} x^{n / 2} \ln ^{k}(x), \\
K & =4 h_{0,0} a_{0}^{2}-\frac{1}{2} P^{2} g_{0}-\frac{1}{2} P^{2} g_{0} \ln (x)+\sum_{n=1}^{\infty} \sum_{k=1}^{n} K_{n, k} x^{n / 2} \ln ^{k}(x), \\
g & =g_{0}+\sum_{n=1}^{\infty} \sum_{k=1}^{n} g_{n, k} x^{n / 2} \ln ^{k}(x) .
\end{aligned}
$$

Most of the coefficients appearing in this expansion are not independent; the independent coefficients correspond either to parameters of the cascading gauge theory or to vacuum expectation values $(\mathrm{VEVs})$ of the operators dual to the fields we are solving 
for. In the KT case there are 3 asymptotic parameters, which we choose to be $g_{0}$, $h_{0,0}$ and $a_{0} . g_{0}$ is related to the dimensionless parameter of the cascading gauge theory, and we will see that one combination of the other parameters is related to the temperature and the other is related to the dynamical scale of the cascading theory. Naively we would expect to have 5 parameters related to VEVs, but in fact there is one relation between the VEVs which is given by the conformal anomaly equation (see [30]), so we are left with four parameters corresponding to VEVs, which we choose to be $\left\{a_{2,0}, g_{2,0}, a_{3,0}, a_{4,0}\right\}$. Note that a VEV appearing at order $x^{n / 2}$ corresponds to an operator which has dimension $2 n$ in the conformal limit of the theory.

Using these 7 parameters we can solve for the coefficients in (3.1) to any order we wish. It turns out that there are no non-zero coefficients at order $\mathcal{O}\left(x^{1 / 2}\right)$. The non-zero coefficients at the following orders are :

- order $\mathcal{O}(x)$ :

$$
\begin{gathered}
h_{2,1}=-\frac{3 g_{0} a_{2,0}}{28 a_{0}^{3}} P^{2}, \quad h_{2,0}=\left(\frac{5 g_{0} a_{2,0}}{28 a_{0}^{3}}-\frac{g_{0}}{16 a_{0}^{2}}-\frac{g_{2,0}}{16 a_{0}^{2}}\right) P^{2}+\frac{3 a_{2,0} h_{0,0}}{7 a_{0}}, \\
b_{2,0}=-\frac{1}{7} a_{2,0}, \quad g_{2,1}=\frac{6 g_{0} a_{2,0}}{7 a_{0}}, \\
K_{2,1}=-\frac{6 g_{0} a_{2,0}}{7 a_{0}} P^{2}, \quad K_{2,0}=\left(\frac{g_{0} a_{2,0}}{a_{0}}-\frac{1}{4} g_{0}-\frac{1}{2} g_{2,0}\right) P^{2}+\frac{24}{7} a_{0} a_{2,0} h_{0,0} .
\end{gathered}
$$

order $\mathcal{O}\left(x^{3 / 2}\right)$

$$
h_{3,0}=\frac{g_{0} a_{3,0}}{60 a_{0}^{3}} P^{2}, \quad b_{3,0}=-\frac{1}{4} a_{3,0}, \quad K_{3,0}=\frac{g_{0} a_{3,0}}{6 a_{0}} P^{2} .
$$

- Order $\mathcal{O}\left(x^{2}\right)$ : using the notation $\delta \equiv 139 P^{2} g_{0}-120 h_{0,0} a_{0}^{2}$, we have

$$
\begin{gathered}
h_{4,3}=-\frac{3 g_{0} a_{2,0}^{2}}{196 a_{0}^{4}} P^{2}, \quad a_{4,2}=-\frac{12 a_{2,0}^{2}}{245 a_{0}}, \quad b_{4,2}=-\frac{12 a_{2,0}^{2}}{245 a_{0}}, \\
h_{4,2}=\frac{1}{\delta}\left\{\left(-\frac{142637 g_{0}^{2} a_{2,0}^{2}}{3920 a_{0}^{4}}-\frac{75 g_{0}^{2} a_{2,0}}{16 a_{0}^{3}}-\frac{5 g_{0}^{2}}{16 a_{0}^{2}}+\frac{75 g_{0}^{2} a_{4,0}}{8 a_{0}^{3}}+\frac{5 g_{2,0}^{2}}{16 a_{0}^{2}}+\frac{139 g_{0} a_{2,0} g_{2,0}}{28 a_{0}^{3}}\right) P^{4}\right. \\
\left.+\frac{1002 g_{0} h_{0,0} a_{2,0}^{2}}{49 a_{0}^{2}} P^{2}-\frac{1440 h_{0,0}^{2} a_{2,0}^{2}}{49}\right\},
\end{gathered}
$$




$$
\begin{aligned}
& h_{4,1}=\frac{1}{\delta}\left\{\left(\frac{95 g_{0}^{2}}{32 a_{0}^{2}}+\frac{230383 g_{0}^{2} a_{2,0}^{2}}{23520 a_{0}^{4}}-\frac{139 g_{0} a_{2,0} g_{2,0}}{24 a_{0}^{3}}-\frac{35 g_{0}^{2} a_{4,0}}{16 a_{0}^{3}}-\frac{7 g_{2,0}^{2}}{96 a_{0}^{2}}-\frac{1423 g_{0}^{2} a_{2,0}}{224 a_{0}^{3}}\right) P^{4}\right. \\
& +\left(\frac{136327 g_{0} h_{0,0} a_{2,0}^{2}}{245 a_{0}^{2}}+\frac{5}{2} g_{0} h_{0,0}-\frac{5 h_{0,0} g_{2,0}^{2}}{g_{0}}-\frac{528 h_{0,0} a_{2,0} g_{2,0}}{7 a_{0}}-\frac{150 g_{0} a_{4,0} h_{0,0}}{a_{0}}\right. \\
& \left.\left.+\frac{570 g_{0} h_{0,0} a_{2,0}}{7 a_{0}}\right) P^{2}+\frac{5160 h_{0,0}^{2} a_{2,0}^{2}}{49}+\frac{5760 a_{0}^{2} h_{0,0}^{3} a_{2,0}^{2}}{49 g_{0} P^{2}}\right\} \\
& h_{4,0}=\frac{1}{\delta}\left\{\left(\frac{31973 g_{0}^{2} a_{2,0}^{2}}{17640 a_{0}^{4}}+\frac{11 g_{0}^{2} a_{4,0}}{4 a_{0}^{3}}+\frac{201 g_{0}^{2} a_{2,0}}{56 a_{0}^{3}}-\frac{219 g_{0}^{2}}{64 a_{0}^{2}}-\frac{139 g_{0} g_{2,0}}{32 a_{0}^{2}}+\frac{695 g_{0} a_{2,0} g_{2,0}}{252 a_{0}^{3}}\right.\right. \\
& \left.-\frac{335 g_{2,0}^{2}}{288 a_{0}^{2}}\right) P^{4}+\left(\frac{35 g_{0} a_{4,0} h_{0,0}}{2 a_{0}}+\frac{67 g_{0} h_{0,0} a_{2,0}}{4 a_{0}}-\frac{167 g_{0} h_{0,0}}{8}-\frac{262231 g_{0} h_{0,0} a_{2,0}^{2}}{2940 a_{0}^{2}}\right. \\
& \left.+\frac{28 h_{0,0} a_{2,0} g_{2,0}}{a_{0}}+\frac{15}{4} g_{2,0} h_{0,0}+\frac{5 h_{0,0} g_{2,0}^{2}}{3 g_{0}}\right) P^{2}+600 a_{0} a_{4,0} h_{0,0}^{2} \\
& +\frac{2104 a_{0} h_{0,0}^{2} a_{2,0} g_{2,0}}{7 g_{0}}-\frac{535356 h_{0,0}^{2} a_{2,0}^{2}}{245}-\frac{2280 a_{0} a_{2,0} h_{0,0}^{2}}{7}+\frac{20 a_{0}^{2} g_{2,0}^{2} h_{0,0}^{2}}{g_{0}^{2}} \\
& \left.-\frac{49536 a_{0}^{2} h_{0,0}^{3} a_{2,0}^{2}}{49 g_{0} P^{2}}\right\} \\
& a_{4,1}=\frac{1}{\delta}\left\{\left(-\frac{12463 g_{0} a_{2,0}^{2}}{70 a_{0}}-\frac{15}{2} g_{0} a_{2,0}-\frac{1}{2} g_{0} a_{0}+15 g_{0} a_{4,0}+\frac{a_{0} g_{2,0}^{2}}{2 g_{0}}\right) P^{2}\right. \\
& \left.+\frac{26688}{245} a_{0} h_{0,0} a_{2,0}^{2}+\frac{48 a_{0}^{2} h_{0,0} a_{2,0} g_{2,0}}{7 g_{0}}-\frac{1152 a_{0}^{3} h_{0,0}^{2} a_{2,0}^{2}}{49 P^{2} g_{0}}\right\} \\
& b_{4,1}=\frac{1}{\delta}\left\{\left(-\frac{7177 g_{0} a_{2,0}^{2}}{490 a_{0}}-\frac{15}{2} g_{0} a_{2,0}-\frac{1}{2} g_{0} a_{0}+15 g_{0} a_{4,0}+\frac{a_{0} g_{2,0}^{2}}{2 g_{0}}\right) P^{2}-\frac{7872 a_{0} h_{0,0} a_{2,0}^{2}}{245}\right. \\
& \left.+\frac{48 a_{0}^{2} h_{0,0} a_{2,0} g_{2,0}}{7 g_{0}}-\frac{1152 a_{0}^{3} h_{0,0}^{2} a_{2,0}^{2}}{49 P^{2} g_{0}}\right\} \\
& b_{4,0}=\frac{1}{\delta}\left\{\left(\frac{6366 g_{0} a_{2,0}^{2}}{245 a_{0}}+6 g_{0} a_{0}+\frac{74}{7} g_{0} a_{2,0}-41 g_{0} a_{4,0}-\frac{6 a_{0} g_{2,0}^{2}}{g_{0}}\right) P^{2}+\frac{126144 a_{0} h_{0,0} a_{2,0}^{2}}{245}\right. \\
& \left.-\frac{576 a_{0}^{2} h_{0,0} a_{2,0} g_{2,0}}{7 g_{0}}-120 a_{0}^{2} a_{4,0} h_{0,0}+\frac{480 a_{2,0} h_{0,0} a_{0}^{2}}{7}+\frac{13824 a_{0}^{3} h_{0,0}^{2} a_{2,0}^{2}}{49 P^{2} g_{0}}\right\} \\
& g_{4,2}=\frac{18 g_{0} a_{2,0}^{2}}{49 a_{0}^{2}}, \quad g_{4,1}=\frac{36 g_{0} a_{2,0}^{2}}{49 a_{0}^{2}}+\frac{3 g_{0} a_{2,0}}{7 a_{0}}+\frac{6 a_{2,0} g_{2,0}}{7 a_{0}}
\end{aligned}
$$




$$
\begin{aligned}
& g_{4,0}=\frac{1}{\delta}\left\{\left(\frac{214407 g_{0}^{2} a_{2,0}^{2}}{980 a_{0}^{2}}+\frac{15 g_{0}^{2}}{4}+\frac{3243 g_{0}^{2} a_{2,0}}{28 a_{0}}-\frac{225 g_{0}^{2} a_{4,0}}{2 a_{0}}+\frac{263 g_{2,0}^{2}}{4}+\frac{139 g_{2,0} g_{0}}{2}\right) P^{2}\right. \\
& +\frac{7200 g_{0} h_{0,0} a_{2,0}^{2}}{49}-\frac{360 a_{0} h_{0,0} a_{2,0} g_{2,0}}{7}-\frac{360 a_{0} g_{0} h_{0,0} a_{2,0}}{7}-60 a_{0}^{2} g_{2,0} h_{0,0} \\
& \left.-\frac{60 a_{0}^{2} g_{2,0}^{2} h_{0,0}}{g_{0}}+\frac{8640 a_{0}^{2} h_{0,0}^{2} a_{2,0}^{2}}{49 P^{2}}\right\} \\
& K_{4,2}=-\frac{12 g_{0} a_{2,0}}{35 a_{0}^{2}} P^{2} \\
& K_{4,1}=\frac{1}{\delta}\left\{\left(-\frac{4701 g_{0}^{2} a_{2,0}}{56 a_{0}}-\frac{9719 g_{0}^{2} a_{2,0}^{2}}{56 a_{0}^{2}}+\frac{195 a_{0}^{2} a_{4,0}}{4 a_{0}}+\frac{13 g_{2,0}^{2}}{8}-\frac{13 g_{0}^{2}}{8}\right.\right. \\
& \left.-\frac{417 g_{0} a_{2,0} g_{2,0}}{14 a_{0}}\right) P^{4}+\left(\frac{360 g_{0} a_{0} a_{2,0} h_{0,0}}{7}+\frac{51096 g_{0} h_{0,0} a_{2,0}^{2}}{245}\right. \\
& \left.\left.+48 a_{0} h_{0,0} a_{2,0} g_{2,0}\right) P^{2}-\frac{12384 a_{2,0}^{2} a_{0}^{2} h_{0,0}^{2}}{49}\right\} \\
& K_{4,0}=\frac{1}{\delta}\left\{\left(-\frac{189 g_{0}^{2}}{16}-\frac{123 g_{0}^{2} a_{2,0}}{56 a_{0}}+\frac{97 g_{0}^{2} a_{4,0}}{4 a_{0}}+\frac{23885 g_{0}^{2} a_{2,0}^{2}}{392 a_{0}^{2}}+\frac{139 g_{0} a_{2,0} g_{2,0}}{14 a_{0}}-\frac{139 g_{0} g_{2,0}}{4}\right.\right. \\
& \left.-\frac{57 g_{2,0}^{2}}{4}\right) P^{4}+\left(\frac{45 g_{0} a_{0}^{2} h_{0,0}}{2}+\frac{283077 g_{0} h_{0,0} a_{2,0}^{2}}{245}+30 a_{0}^{2} g_{2,0} h_{0,0}-390 g_{0} a_{0} a_{4,0} h_{0,0}\right. \\
& \left.+\frac{2973 g_{0} a_{0} a_{2,0} h_{0,0}}{7}+\frac{240 a_{0} h_{0,0} a_{2,0} g_{2,0}}{7}\right) P^{2}-\frac{1440 a_{0}^{3} h_{0,0}^{2} a_{2,0} g_{2,0}}{7 g_{0}} \\
& \left.-\frac{1440 a_{0}^{3} h_{0,0}^{2} a_{2,0}}{7}+\frac{35712 a_{2,0}^{2} a_{0}^{2} h_{0,0}^{2}}{49}\right\} \text {. }
\end{aligned}
$$

\subsection{The IR boundary conditions}

Next, we discuss the behavior of solutions to (2.8)-(2.12) near the horizon, $x \rightarrow 1$. Introducing a near-horizon coordinate

$$
y \equiv 1-x
$$

we find that in order for the solutions (2.4) to have a non-singular Schwarzschild horizon, the functions $\left\{h, f_{2}, f_{3}, g, K\right\}$ must all be even functions of $y$ with a good Taylor 
series expansion around $y=0$ :

$$
\begin{aligned}
h & =\sum_{n=0}^{\infty} h_{n}^{h} y^{2 n}, & f_{2} & =\sum_{n=0}^{\infty} a_{n}^{h} y^{2 n}, \quad f_{3}=\sum_{n=0}^{\infty} b_{n}^{h} y^{2 n}, \\
K & =\sum_{n=0}^{\infty} k_{n}^{h} y^{2 n}, & g & =\sum_{n=0}^{\infty} g_{n}^{h} y^{2 n} .
\end{aligned}
$$

When one solves the equations of motion perturbatively in $y$, one finds that the solutions are labeled by six independent parameters, which one can choose to be $\left\{h_{0}^{h}, a_{0}^{h}, b_{0}^{h}, k_{0}^{h}, g_{0}^{h}, a_{1}^{h}\right\}$. Naively one might think that all five equations of motion (2.8)(2.12) would have one normalizable mode and one non-normalizable mode near the horizon, so that requiring a regular solution will set the coefficients of the non-normalizable modes to zero and leave us with 5 parameters. However, it turns out that for one combination of the equations both modes are normalizable near the horizon, leading to the extra parameter; this is related to a scaling symmetry of the geometry (2.4) which we will discuss in the next subsection, which implies that one combination of parameters is not determined before choosing a scale ${ }^{7}$.

Using these 6 parameters we can solve for the coefficients in (3.19) to any order we wish. Using the notation $\delta^{h} \equiv 8 h_{0}^{h}\left(a_{0}^{h}\right)^{2}-P^{2} g_{0}^{h}$, we have

- order $\mathcal{O}\left(y^{2}\right)$ :

$$
\begin{aligned}
& h_{1}^{h}=\frac{1}{\delta^{h}}\left\{8\left(a_{0}^{h}\right)^{2}\left(h_{0}^{h}\right)^{2}-\frac{\left(k_{0}^{h}\right)^{2}}{2\left(b_{0}^{h}\right)^{2}}-\frac{a_{1}^{h}\left(k_{0}^{h}\right)^{2}}{\left(b_{0}^{h}\right)^{2} a_{0}^{h}}-\left(\frac{3}{2} h_{0}^{h} g_{0}^{h}+\frac{h_{0}^{h} g_{0}^{h} a_{1}^{h}}{a_{0}^{h}}\right) P^{2}\right\}, \\
& b_{1}^{h}=\frac{1}{\delta^{h}}\left\{\frac{1}{2} g_{0}^{h} b_{0}^{h} P^{2}-2 h_{0}^{h} b_{0}^{h}\left(3\left(a_{0}^{h}\right)^{2}-3 a_{0}^{h} b_{0}^{h}+2 a_{0}^{h} a_{1}^{h}-6 a_{1}^{h} b_{0}^{h}\right)\right\}, \\
& k_{1}^{h}=\frac{1}{\delta^{h}}\left\{\frac{P^{2} g_{0}^{h} k_{0}^{h}\left(a_{0}^{h}+2 a_{1}^{h}\right)}{a_{0}^{h}}\right\}, \quad g_{1}^{h}=\frac{1}{\delta^{h}}\left\{\frac{\left(g_{0}^{h}\right)^{2} P^{2}\left(a_{0}^{h}+2 a_{1}^{h}\right)}{2 a_{0}^{h}}\right\} .
\end{aligned}
$$

- order $\mathcal{O}\left(y^{4}\right)$ :

$$
\begin{aligned}
& k_{2}^{h}=\frac{1}{\left(\delta^{h}\right)^{2}}\left\{-\frac{g_{0}^{h} P^{2} k_{0}^{h}\left(a_{0}^{h}+2 a_{1}^{h}\right)^{2}}{2\left(a_{0}^{h}\right)^{2}}\left(4 h_{0}^{h}\left(a_{0}^{h}\right)^{2}-12 h_{0}^{h} a_{0}^{h} b_{0}^{h}-g_{0}^{h} P^{2}\right)\right\}, \\
& g_{2}^{h}=\frac{1}{\left(\delta^{h}\right)^{2}}\left\{-\frac{\left(g_{0}^{h}\right)^{2} P^{2}\left(a_{0}^{h}+2 a_{1}^{h}\right)^{2}}{4\left(a_{0}^{h}\right)^{2}}\left(2 h_{0}^{h}\left(a_{0}^{h}\right)^{2}-6 h_{0}^{h} a_{0}^{h} b_{0}^{h}-g_{0}^{h} P^{2}\right)\right\},
\end{aligned}
$$

\footnotetext{
${ }^{7}$ Such a scaling symmetry in geometries with translationally invariant horizons was also noticed in $[34]$.
} 


$$
\begin{aligned}
h_{2}^{h} & =\frac{1}{\left(\delta^{h}\right)^{2}}\left\{\frac{4 h_{0}^{h} a_{0}^{h}}{\left(b_{0}^{h}\right)^{2}}\left(16\left(b_{0}^{h}\right)^{2}\left(a_{0}^{h}\right)^{3}\left(h_{0}^{h}\right)^{2}-a_{0}^{h}\left(k_{0}^{h}\right)^{2}-2 a_{1}^{h}\left(k_{0}^{h}\right)^{2}\right)+\frac{h_{0}^{h}\left(g_{0}^{h}\right)^{2} P^{4}\left(3 a_{0}^{h}+2 a_{1}^{h}\right)}{2 a_{0}^{h}}\right. \\
- & \frac{g_{0}^{h} P^{2}}{4\left(a_{0}^{h}\right)^{2}\left(b_{0}^{h}\right)^{2}}\left(78\left(h_{0}^{h}\right)^{2}\left(b_{0}^{h}\right)^{2}\left(a_{0}^{h}\right)^{4}+6\left(h_{0}^{h}\right)^{2}\left(a_{0}^{h}\right)^{3}\left(b_{0}^{h}\right)^{3}-8\left(h_{0}^{h}\right)^{2}\left(a_{0}^{h}\right)^{2}\left(a_{1}^{h}\right)^{2}\left(b_{0}^{h}\right)^{2}\right. \\
+ & 24\left(h_{0}^{h}\right)^{2}\left(a_{1}^{h}\right)^{2}\left(b_{0}^{h}\right)^{3} a_{0}^{h}+24\left(h_{0}^{h}\right)^{2}\left(a_{0}^{h}\right)^{3} a_{1}^{h}\left(b_{0}^{h}\right)^{2}+24\left(h_{0}^{h}\right)^{2}\left(a_{0}^{h}\right)^{2} a_{1}^{h}\left(b_{0}^{h}\right)^{3}-\left(k_{0}^{h}\right)^{2}\left(a_{0}^{h}\right)^{2} \\
+ & \left.\left.4\left(k_{0}^{h}\right)^{2}\left(a_{1}^{h}\right)^{2}\right)\right\}, \\
& a_{2}^{h}=\frac{1}{\left(\delta^{h}\right)^{2}}\left\{-12\left(h_{0}^{h}\right)^{2} a_{0}^{5}+12 b_{0}^{h}\left(h_{0}^{h}\right)^{2}\left(a_{0}^{h}\right)^{4}+16\left(h_{0}^{h}\right)^{2}\left(a_{0}^{h}\right)^{4} a_{1}^{h}+48\left(h_{0}^{h}\right)^{2}\left(a_{0}^{h}\right)^{3} a_{1}^{h} b_{0}^{h}\right. \\
& +48\left(h_{0}^{h}\right)^{2}\left(a_{0}^{h}\right)^{3}\left(a_{1}^{h}\right)^{2}+48\left(h_{0}^{h}\right)^{2}\left(a_{0}^{h}\right)^{2} b_{0}^{h}\left(a_{1}^{h}\right)^{2}+\left(\frac{7}{2}\left(a_{0}^{h}\right)^{3} h_{0}^{h} g_{0}^{h}-\frac{3}{2} b_{0}^{h} g_{0}^{h} h_{0}^{h}\left(a_{0}^{h}\right)^{2}\right. \\
& \left.-2 h_{0}^{h}\left(a_{0}^{h}\right)^{2} a_{1}^{h} g_{0}^{h}-6 h_{0}^{h} a_{0}^{h} a_{1}^{h} b_{0}^{h} g_{0}^{h}-10 h_{0}^{h} a_{0}^{h}\left(a_{1}^{h}\right)^{2} g_{0}^{h}-6 h_{0}^{h}\left(a_{1}^{h}\right)^{2} g_{0}^{h} b_{0}^{h}\right) P^{2} \\
& \left.+\left(-\frac{3}{8} a_{0}^{h}\left(g_{0}^{h}\right)^{2}-\frac{1}{2}\left(g_{0}^{h}\right)^{2} a_{1}^{h}\right)^{4}\right\}, \\
& \left.\left.+12 a_{0}^{h} a_{1}^{h} b_{0}^{h}+8 a_{0}^{h}\left(a_{1}^{h}\right)^{2}-12\left(a_{1}^{h}\right)^{2} b_{0}^{h}\right)-\frac{1}{8}\left(g_{0}^{h}\right)^{2} b_{0}^{h} P^{4}\right\} . \\
& b_{2}^{h}=\frac{1}{\left(\delta^{h}\right)^{2}}\left\{( h _ { 0 } ^ { h } ) ^ { 2 } \left(-45 b_{0}^{h}\left(a_{0}^{h}\right)^{3}+16\left(a_{0}^{h}\right)^{3} a_{1}^{h}+45\left(b_{0}^{h}\right)^{2}\left(a_{0}^{h}\right)^{2}-132\left(a_{0}^{h}\right)^{2} a_{1}^{h} b_{0}^{h}\right.\right. \\
& \left.180\left(b_{0}^{h}\right)^{2} a_{0}^{h} a_{1}^{h}-84 a_{0}^{h} b_{0}^{h}\left(a_{1}^{h}\right)^{2}+180\left(b_{0}^{h}\right)^{2}\left(a_{1}^{h}\right)^{2}\right) b_{0}^{h}+\frac{h_{0}^{h} g_{0}^{h} P^{2} b_{0}^{h}}{4 a_{0}^{h}}\left(6\left(a_{0}^{h}\right)^{3}+9 b_{0}^{h}\left(a_{0}^{h}\right)^{2}\right.
\end{aligned}
$$

\section{Mapping of parameters to the field theory}

\subsection{Translation to the parametrization of [30]}

In this section we wish to understand the physical meaning of the 3 parameters which we used in the previous section to parameterize our theory (in the UV) $-h_{0,0}, a_{0}$ and $g_{0}$. As we mentioned, $g_{0} P$ is the dimensionless parameter of the cascading theory (which must be large for the gravity approximation to be valid), while $h_{0,0}$ and $a_{0}$ are related to the scale of cascading theory and to the temperature. Note that our ansatz (2.4) is invariant under a scaling symmetry taking

$$
(t, \vec{x}) \rightarrow \lambda^{-2}(t, \vec{x}), \quad h \rightarrow \lambda^{-2} h, \quad f_{2} \rightarrow \lambda f_{2}, \quad f_{3} \rightarrow \lambda f_{3}
$$


and leaving all other functions in our solution (as well as the coordinate $x$ ) invariant. In terms of our asymptotic parameters, this scaling transformation leaves $h_{0,0} a_{0}^{2}$ invariant, meaning that this combination is a function of the dimensionless parameter of our theory, which is the ratio between the temperature and some scale $\Lambda$ which characterizes the cascading theory. We can choose this scale $\Lambda$ to be, say, the mass of the lightest glueball, or the square root of the string tension. We will find it more convenient to use a different definition of $\Lambda$ which will be described below.

The parametrization (2.4) we used above for the solution breaks down at zero temperature, since it assumes the existence of a horizon. In order to understand which combinations of our parameters depend on the temperature and which do not it is convenient to switch to a different parametrization of the geometry, which is valid also at low temperatures : an example of such a parametrization is given by [30]

$$
\begin{aligned}
d s_{10}^{2}= & \hat{h}^{-1 / 2} \rho^{-2}\left(-\hat{f}^{2} d t^{2}+d x_{1}^{2}+d x_{2}^{2}+d x_{3}^{2}\right)+\hat{h}^{1 / 2} \rho^{-2}(d \rho)^{2} \\
& +\hat{h}^{1 / 2} \hat{f}_{2}\left(e_{\psi}^{2}\right)+\hat{h}^{1 / 2} \hat{f}_{3} \sum_{a=1}^{2}\left(e_{\theta_{a}}^{2}+e_{\phi_{a}}^{2}\right),
\end{aligned}
$$

where $\left\{\hat{h}, \hat{f}_{2}, \hat{f}_{3}, \hat{f}\right\}$ are functions of $\rho$. In this parametrization the supersymmetric zero temperature solution is characterized by two parameters: the value of the string coupling $\hat{g}_{0}$, and the coefficient of the warp factor $\hat{h}_{0,0}$; in terms of these parameters we can write the asymptotic solution for small $\rho$ as

$$
\begin{aligned}
& \hat{h}=\hat{h}_{0,0}-\frac{1}{2} \hat{g}_{0} P^{2} \ln (\rho), \quad \hat{K}=4 \hat{h}_{0,0}-\frac{1}{2} \hat{g}_{0} P^{2}-2 \hat{g}_{0} P^{2} \ln (\rho), \\
& \hat{g}=\hat{g}_{0}, \quad \hat{f}=\hat{f}_{2}=\hat{f}_{3}=1 .
\end{aligned}
$$

Note that the ansatz (4.2) is invariant under a joint rescaling of the $x, t$ coordinates and the $\rho$ coordinate; such a rescaling leads to a constant shift in $\hat{h}_{0,0}$. Thus, we can think of $\hat{h}_{0,0}$ as determining the scale of the cascading theory; note that this is independent of the temperature, since in the parametrization (4.2) all IR effects (including the effects of the temperature) are suppressed by powers of $\rho$.

We would like to match (4.3) with the asymptotic solution (3.1) used above. We require that as $\rho \rightarrow 0$ (and correspondingly $x \rightarrow 0$ ) all the corresponding warp factors in the metric should agree to leading order, i.e.,

$$
\begin{aligned}
& \lim _{\{\rho, x\} \rightarrow 0} \frac{\rho^{-2} \hat{f}(\rho)^{2} \hat{h}(\rho)^{-1 / 2}}{(1-x)^{2}\left(2 x-x^{2}\right)^{-1 / 2} h(x)^{-1 / 2}}=1, \quad \lim _{\{\rho, x\} \rightarrow 0} \frac{\hat{h}(\rho)^{1 / 2} \hat{f}_{2}(\rho)}{h(x)^{1 / 2} f_{2}(x)}=1, \\
& \lim _{\{\rho, x\} \rightarrow 0} \frac{\hat{h}(\rho)^{-1 / 2} \hat{f}_{3}(\rho)}{h(x)^{-1 / 2} f_{3}(x)}=1, \quad \lim _{\{\rho, x\} \rightarrow 0} \frac{\hat{g}(\rho)}{g(x)}=1, \quad \lim _{\{\rho, x\} \rightarrow 0} \frac{\hat{K}(\rho)}{K(x)}=1 .
\end{aligned}
$$


This matching uniquely identifies:

$$
x=\frac{1}{2} a_{0}^{2} \rho^{4}+\text { higher orders }, \quad g_{0}=\hat{g}_{0}, \quad h_{0,0} a_{0}^{2}=\hat{h}_{0,0}+\frac{1}{8} P^{2} \hat{g}_{0} \ln \left(\frac{a_{0}^{2}}{2}\right) .
$$

\subsection{Expectation values in the black hole background}

In order to proceed, we would like to compute the expectation values of various operators in the cascading theories in terms of our parameters; in particular we want to compute the expectation value of the stress-energy tensor and of scalar operators which have dimension four when $P \rightarrow 0$. The expectation values of these operators were evaluated in [30] using the coefficients appearing in the expansion in powers of $\rho$ of the functions appearing in (4.2), up to order $\rho^{4}$. In order to recycle those results we need to translate our boundary expansion of the previous section to the one of [30], namely to write the leading terms of the expansion of [30] in terms of our parameters $\left\{h_{0,0}, a_{0}, g_{0}, a_{2,0}\right\}$, as we did for the zeroth order terms in (4.5) ${ }^{8}$.

When we do the matching we have some freedom, since in the parametrization (4.2) there is a freedom of performing diffeomorphisms of $\rho$ depending on higher powers of $\rho$, that only affect the higher order terms in the expansion. Of course this freedom does not affect the eventual expectation values. We will fix this freedom by making an explicit choice for $x$ as a function of $\rho$ to order $\mathcal{O}\left(\rho^{8}\right)$, given by :

$$
x=\frac{1}{2} \rho^{4} a_{0}^{2}-\rho^{8} a_{0}^{3}\left(\frac{5}{24} a_{0}+\frac{1}{14} a_{2,0}\right)+\mathcal{O}\left(\rho^{10}\right) .
$$

We can now identify all the terms in the expansion of [30] using our expansion of the previous section. Translating (4.5) to the notation of [30], we find at order $\rho^{0}$

$$
\begin{aligned}
& p_{0}=g_{0}, \quad K_{0}=4 h_{0,0} a_{0}^{2}-\frac{1}{2} P^{2} g_{0}-\frac{1}{2} P^{2} g_{0} \ln \left(\frac{a_{0}^{2}}{2}\right), \\
& G_{i j}^{(0)}=\eta_{i j}=\operatorname{diag}(-1,1,1,1) .
\end{aligned}
$$

All the coefficients in [30] at order $\rho^{2}$ vanish, while the independent parameters appearing at order $\rho^{4}$ are given by (again in the notation of [30])

$$
\begin{aligned}
& a^{(4,1)}=0, \quad a^{(4,2)}=0, \quad a^{(4,3)}=0, \quad G_{t t}^{(4,0)}=a_{0}^{2}, \quad G_{x_{i} x_{i}}^{(4,0)}=0, \\
& p^{(4,0)}=\frac{a_{0}^{2} g_{2,0}}{2 g_{0}}+\frac{3}{7} a_{0} a_{2,0} \ln \left(\frac{a_{0}^{2}}{2}\right), \quad a^{(4,0)}=\frac{4}{7} a_{0} a_{2,0}+\frac{1}{3} a_{0}^{2}, \quad b^{(4,0)}=\frac{1}{3} a_{0}^{2} .
\end{aligned}
$$

\footnotetext{
${ }^{8}$ Note that the parameters $\left\{a_{3,0}, a_{4,0}\right\}$ only show up at orders $\mathcal{O}\left(\rho^{6}\right)$ and $\mathcal{O}\left(\rho^{8}\right)$, respectively, so they do not affect the expectation values of these operators.
} 
The one-point function of the stress energy tensor is given by [30]

$$
8 \pi G_{5}\left\langle T_{i j}\right\rangle=-\frac{1}{2} G_{i j}^{(0)} G_{a}^{(4,0) a}+2 G_{i j}^{(4,0)}+\frac{3}{2} G_{i j}^{(0)}\left(b^{(4,0)}-a^{(4,0)}\right),
$$

where $G_{5}$ is the five dimensional Newton's constant obtained after we do the dimensional reduction on $T^{1,1}$ (see [30]). In the normalizations that we are using, $G_{5}$ is related to the ratio $P / M$ (using the careful analysis of [35]) by $G_{5}=8 \pi^{3} P^{4} / 81 M^{4}$. Using (4.9) we obtain that the energy density $\mathcal{E}$ and the pressure $\mathcal{P}$ are given in terms of our parameters by

$$
\begin{aligned}
& \mathcal{E} \equiv\left\langle T_{t t}\right\rangle=\frac{1}{8 \pi G_{5}}\left(\frac{3}{2} a_{0}^{2}+\frac{6}{7} a_{0} a_{2,0}\right), \\
& \mathcal{P} \equiv\left\langle T_{x_{i} x_{i}}\right\rangle=\frac{1}{8 \pi G_{5}}\left(\frac{1}{2} a_{0}^{2}-\frac{6}{7} a_{0} a_{2,0}\right) .
\end{aligned}
$$

Since we do not have any chemical potentials, the free energy density $\mathcal{F}$ is

$$
\mathcal{F}=-\mathcal{P}=\frac{1}{8 \pi G_{5}}\left(\frac{6}{7} a_{0} a_{2,0}-\frac{1}{2} a_{0}^{2}\right) .
$$

The expectation values of the remaining two scalar operators which have dimension 4 when $P=0$ are (using their normalization defined in [30])

$$
\begin{aligned}
\left\langle\mathcal{O}_{K_{0}}\right\rangle & =\frac{24 a_{0} a_{2,0}}{7 P^{2} g_{0}}, \\
\left\langle\mathcal{O}_{p_{0}}\right\rangle & =2 \frac{a_{0}^{2} g_{2,0}}{g_{0}^{2}}+\frac{12 a_{0} a_{2,0}}{7 g_{0}}\left(1+\ln \left(\frac{a_{0}^{2}}{2}\right)\right) .
\end{aligned}
$$

Note that in general curved backgrounds there was an ambiguity in some of the onepoint correlation functions computed in [30], but there is no such ambiguity when the asymptotic four dimensional metric is flat (as in our case).

\subsection{The basic thermodynamic relation}

The equations above tell us, using the asymptotic values of the fields, that

$$
s T=\mathcal{E}-\mathcal{F}=\mathcal{E}+\mathcal{P}=\frac{a_{0}^{2}}{4 \pi G_{5}}
$$

in the cascading background, where $s$ is the entropy density. On the other hand, we can also compute the entropy density and temperature directly at the horizon in terms of the horizon parameters $\left\{h_{0}^{h}, a_{0}^{h}, b_{0}^{h}, k_{0}^{h}, g_{0}^{h}, a_{1}^{h}\right\}$ :

$$
s=\frac{\left(a_{0}^{h}\right)^{1 / 2}\left(b_{0}^{h}\right)^{2}\left(h_{0}^{h}\right)^{1 / 2}}{4 G_{5}}, \quad T=\frac{1}{4 \pi h_{0}^{h} b_{0}^{h}} \sqrt{\frac{2\left(8 h_{0}^{h}\left(a_{0}^{h}\right)^{2}-g_{0}^{h} P^{2}\right)}{a_{0}^{h}+2 a_{1}^{h}}} .
$$


At first sight the previous two equations seem to give a non-trivial relation between some of our UV parameters and some of the IR parameters related to the expansion near the horizon. However, it turns out that $s T$ is a renormalization group flow invariant in supergravity black brane geometries without a chemical potential $[36,37]$, so this relation is trivially satisfied in any solution of our equations of motion.

To simplify notations we rewrite the metric (2.4) as

$$
d s_{10}^{2}=-c_{1}^{2} d t^{2}+c_{2}^{2}\left(d x_{1}^{2}+d x_{2}^{2}+d x_{3}^{3}\right)+c_{3}^{2}(d x)^{2}+c_{4}^{2}\left(e_{\psi}^{2}\right)+c_{5}^{2} \sum_{a=1}^{2}\left(e_{\theta_{a}}^{2}+e_{\phi_{a}}^{2}\right),
$$

where $c_{i}=c_{i}(x)$ can be identified by comparing (2.4) and (4.15). Now, from the relation between components of the Ricci tensor

$$
R_{x_{1}}^{x_{1}}=R_{t}^{t}
$$

we have a constraint ${ }^{9}$

$$
\frac{c_{2}^{4} c_{4} c_{5}^{4}}{c_{3}}\left(\frac{c_{1}}{c_{2}}\right)^{\prime}=\text { constant }
$$

Evaluating the left-hand side of (4.17) near the horizon, using the standard relations between the area of the horizon and the entropy and between the surface gravity of the horizon and the temperature, we have

$$
\lim _{x \rightarrow 1_{-}} \frac{c_{2}^{4} c_{4} c_{5}^{4}}{c_{3}}\left|\left(\frac{c_{1}}{c_{2}}\right)^{\prime}\right|=8 \pi G_{5} s T .
$$

On the other hand, evaluating the left-hand side of (4.17) near the boundary and using the asymptotic solution (3.1) we find

$$
\lim _{x \rightarrow 0_{+}} \frac{c_{2}^{4} c_{4} c_{5}^{4}}{c_{3}}\left|\left(\frac{c_{1}}{c_{2}}\right)^{\prime}\right|=\lim _{x \rightarrow 0_{+}} \frac{h^{1 / 4} f_{2}^{1 / 2} f_{3}^{2}}{\left(2 x-x^{2}\right) G_{x x}^{1 / 2}}=2 a_{0}^{2} .
$$

Thus, our equation (4.13) follows in a straightforward way from the equations of motion.

\section{The numerical procedure}

\subsection{Reducing the number of parameters}

Before we begin the numerical solution of the equations, we can use the symmetries of the problem to get rid of some of our parameters. First, as mentioned above, the

\footnotetext{
${ }^{9}$ Equation (4.17) can also be directly derived from (2.8)-(2.12).
} 
parameters $h_{0,0}$ and $a_{0}$ are not scale invariant, but only the combination $h_{0,0} a_{0}^{2}$, which is a function of the temperature divided by the dynamical scale. We will choose as our parameter which is related to the dimensionless temperature the combination $k_{s}$ defined by

$$
P^{2} g_{0} k_{s} \equiv 4 h_{0,0} a_{0}^{2}-\frac{1}{2} P^{2} g_{0}
$$

Equation (4.5) now tells us that $\left[k_{s}-\ln \left(a_{0}^{2} / 2\right) / 2\right]$ is independent of the temperature (it depends only on the dynamical scale of the cascading theory). Thus, we can choose to define the scale $\Lambda$ of this theory by a relation of the form

$$
k_{s} \equiv \frac{1}{2} \ln \left(\frac{a_{0}^{2}}{\Lambda^{4}}\right)=\frac{1}{2} \ln \left(\frac{4 \pi G_{5} s T}{\Lambda^{4}}\right) .
$$

Using the expressions for the high temperature entropy density of the theory computed in $[11,10]$, we see that at high temperatures $k_{s} \simeq(1 / 2) \ln \left(T^{4} / \Lambda^{4}\right)$, with corrections scaling as $\ln (\ln (T / \Lambda))$. We will use $k_{s}$ instead of the temperature as our basic dimensionless parameter, and use (5.2) to translate between $k_{s}$ and $T / \Lambda$.

Having understood this relation, we can now use the scaling symmetry (4.1) to set $a_{0}=1$, or, equivalently, use the fact that the solution to the equations of motion depends on the $7 \mathrm{UV}$ parameters $\left\{g_{0}, h_{0,0}, a_{0}, a_{2,0}, g_{2,0}, a_{3,0}, a_{4,0}\right\}$ that we used in our expansion only through the six invariant combinations

$$
\left\{g_{0}, k_{s}, \hat{a}_{2,0} \equiv \frac{a_{2,0}}{a_{0}}, \hat{a}_{3,0} \equiv \frac{a_{3,0}}{a_{0}}, \hat{a}_{4,0} \equiv \frac{a_{4,0}}{a_{0}}, g_{2,0}\right\} .
$$

Recall also that we are solving the theory in the supergravity approximation, which includes only the leading order terms both in the $g_{s}$ expansion and in the curvature $\left(\alpha^{\prime}\right)$ expansion. When we neglect $g_{s}$ corrections, the action (and the equations of motion we wrote) does not depend separately on $P^{2}$ and $g$ but only on the combination $P^{2} g$. We can thus set $g_{0}=1$, and recall that whenever we have a factor of $P^{2}$ we really mean $P^{2} g_{0}$. Furthermore, when we neglect $\alpha^{\prime}$ corrections, the action is multiplied by a constant when we rescale the ten dimensional metric by a constant factor (and rescale the $p$-forms accordingly), so that the equations of motion are left invariant; this transformation acts on our variables as

$$
h \rightarrow \lambda^{-2} h, \quad f_{2,3} \rightarrow \lambda^{2} f_{2,3}, \quad K \rightarrow \lambda^{2} K, \quad g \rightarrow g,
$$

and it changes $P$ by $P \rightarrow \lambda P$. We can use this transformation to relate the solutions for different values of $P$ (as long as we are in the supergravity approximation). Thus, 
we will perform the numerical analysis for $P=1$, and we can use (5.4) to obtain the solutions for any other value of $P$.

As a test of our numerics, we can check if it reproduces the solution at high temperatures which can be computed perturbatively (as was done at leading order in [10], and at higher orders in the appendix). This computation implies that the correct solution should obey at large $k_{s}$ (A.35)-(A.38):

$$
\begin{aligned}
\hat{a}_{2,0} & =\frac{7}{12} \frac{1}{k_{s}}-\frac{7}{24} \ln (2) \frac{1}{k_{s}^{2}}+\mathcal{O}\left(k_{s}^{-3}\right) \\
\hat{a}_{3,0} & =\frac{4}{5} \lambda_{3}^{[2]} \frac{1}{k_{s}}+\left(\left(\frac{2}{15}-\frac{2}{5} \ln (2)\right) \lambda_{3}^{[2]}+\frac{4}{5} \lambda_{3}^{[4]}\right) \frac{1}{k_{s}^{2}}+\mathcal{O}\left(k_{s}^{-3}\right) \\
\hat{a}_{4,0} & =\left(\frac{\ln (2)}{30}+\frac{1021}{1800}\right) \frac{1}{k_{s}}+\left(\frac{167809}{108000}-\frac{(\ln (2))^{2}}{360}-\frac{781}{1200} \ln (2)+\eta_{4}^{[4]}\right) \frac{1}{k_{s}^{2}}+\mathcal{O}\left(k_{s}^{-3}\right), \\
g_{2,0} & =\left(-\frac{1}{2}+\frac{1}{2} \ln (2)\right) \frac{1}{k_{s}}+\left(\frac{1}{4} \ln (2)-\frac{1}{4}(\ln (2))^{2}+\zeta_{2}^{[4]}\right) \frac{1}{k_{s}^{2}}+\mathcal{O}\left(k_{s}^{-3}\right),
\end{aligned}
$$

where the values of the various constants appear in the appendix, and we used the high-temperature relation between the value of $K$ at the horizon, which we denote by $K_{\star}$, and our dimensionless parameter $k_{s}$ :

$$
K_{\star}=P^{2} \hat{g}_{0}\left(k_{s}+\frac{1}{2} \ln (2)+\mathcal{O}\left(k_{s}^{-1}\right)\right) .
$$

\subsection{Our numerical method}

As described above, for a given value of the temperature (or of $k_{s}$ ) we have four parameters controlling the behavior of our solutions near the UV. What we need to do is to find for which value of these four parameters the solution is regular near the horizon, and this will determine the correct vacuum expectation values for this value of the temperature. The most naive way to proceed would be to go over all possible values of these parameters, use these values to determine the solution near the boundary, integrate the equations of motion up to $x=1$, and see if the solution there is regular or not. Unfortunately, we cannot integrate the equations analytically but only numerically, and when we integrate the equations near the horizon, numerical errors always generate modes that blow up at the horizon, so we cannot really obtain solutions that are regular at the horizon in this way.

One alternative might be to perform the integration in the opposite direction - start from a general solution near the horizon, integrate the equations to the boundary, and 
see for which values of our near-horizon parameters we find a regular solution at the boundary (with the correct KT asymptotics). However, this suffers from the same problem, that numerical errors generate modes that grow near the boundary.

Thus, we are led to a procedure where we integrate the equations both from the boundary and from the horizon towards the middle of the interval $x=0.5$, and attempt to match a solution that we get by integrating from the boundary with a solution that we get by integrating from the horizon. After setting $g_{0}=1$, for a given value of $k_{s}$, the UV behaviour (3.1) is determined by 4 parameters (related to operator VEVs) (5.3)

$$
\left\{\hat{a}_{2,0}, \hat{a}_{3,0}, \hat{a}_{4,0}, g_{2,0}\right\}
$$

The IR behaviour (3.19) is determined by 6 horizon parameters

$$
\left\{h_{0}^{h}, a_{0}^{h}, a_{1}^{h}, b_{0}^{h}, k_{0}^{h}, g_{0}^{h}\right\}
$$

Matching a UV solution and an IR solution to (2.8)-(2.12) at $x=0.5$ implies 10 constraints ( 5 for matching the values of the functions, and 5 for matching their derivative). Notice that we have precisely the same number of constraints as necessary to uniquely determine all the UV and IR parameters ((5.7) and (5.8)) for a given value of $k_{s}$.

Since both the boundary $x=0$ and the horizon $x=1$ are singular points of the differential equations (2.8)-(2.12), we integrate the differential equations (2.8)-(2.12) from $x=0.01$ (for the boundary integration) and from $y=0.01$ (for the horizon integration). In the former case the initial conditions are specified by the asymptotic expansion (3.1) which we developed to order $x^{9 / 2}$ (inclusive); in the case of the horizon integration the initial conditions are specified by the asymptotic expansion (3.19) to order $y^{10}$ (inclusive). The coefficients of these asymptotic expansions generalize the results presented in section 3, and are available from the authors upon request. The mismatch between the boundary and the horizon integrations is encoded in the 'mismatch vector' $\vec{v}_{\text {mismatch }}$, defined by

$$
\begin{aligned}
\vec{v}_{\text {mismatch }}= & \left(h_{b}-h_{h}, h_{b}^{\prime}+h_{h}^{\prime}, f_{2, b}-f_{2, h}, f_{2, b}^{\prime}+f_{2, h}^{\prime}, f_{3, b}-f_{3, h}, f_{3, b}^{\prime}+f_{3, h}^{\prime},\right. \\
& \left.K_{b}-K_{h}, K_{b}^{\prime}+K_{h}^{\prime}, g_{b}-g_{h}, g_{b}^{\prime}+g_{h}^{\prime}\right)\left.\right|_{x=y=0.5},
\end{aligned}
$$

where the subscripts ${ }_{h}$ or ${ }_{b}$ correspond to functions $\left\{h, f_{2}, f_{3}, K, g\right\}$ integrated from the horizon or boundary, respectively, and the prime denotes derivatives with respect to $x$ 


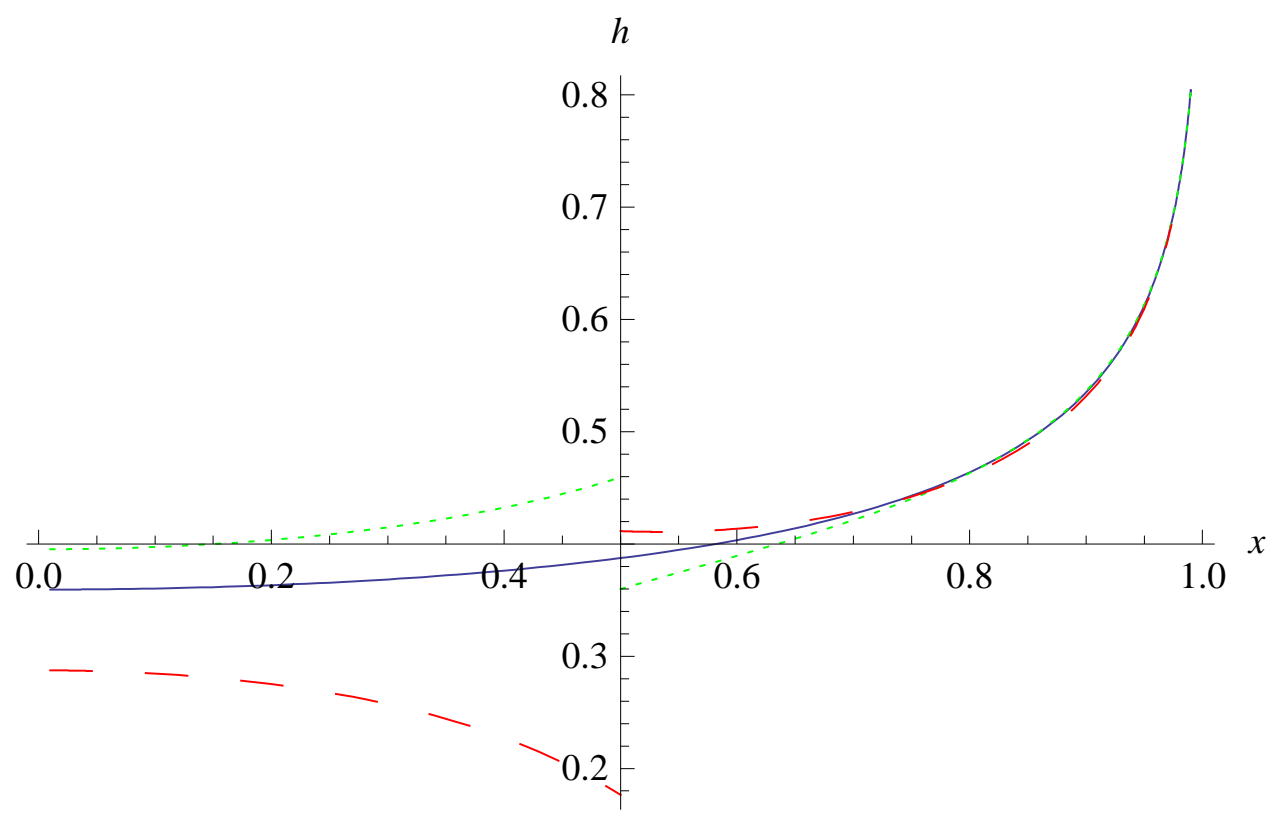

Figure 1: Mismatch of $h_{b}(x)$ (for $x<0.5$ ) and $h_{h}(y \equiv 1-x)$ (for $x>0.5$ ) for different values of the parameters, for $k_{s}=0.4$. The solid (blue) curves correspond to "correct" values of the parameters (5.7) and (5.8), with $\left\|\vec{v}_{\text {mismatch }}\right\| \approx 9 \times 10^{-6}$. The dotted (green) curves corresponding to all values of parameters $10 \%$ larger than the correct ones, produce $\left\|\vec{v}_{\text {mismatch }}\right\| \approx 3 \times 10^{-1}$. The dashed (red) curves correspond to all values of parameters $20 \%$ smaller than the correct ones, giving $\left\|\vec{v}_{\text {mismatch }}\right\| \approx 8 \times 10^{-1}$.

or $y$. The UV parameters (5.7) and the IR parameters (5.8) are tuned to ensure that

$$
\left\|\vec{v}_{\text {mismatch }}\right\|<10^{-5} \text {. }
$$

An illustration of the integration as a function of the UV and IR parameters is presented in figure 1.

We performed the numerical integration using Wolfram Mathematica $^{\circledR} 6$ with 40 digit precision, to ensure sensitivity to the irrelevant operator parameters $\hat{a}_{3,0}$ and $\hat{a}_{4,0}$.

\subsection{The numerical results}

We present the numerical results for the UV (5.7) and the IR (5.8) parameters as a function of $k_{s}$ in two regimes ${ }^{10}$ :

\footnotetext{
${ }^{10}$ The IR parameters are presented only for small values of $k_{s}$. Additional data are available from the authors upon request.
} 

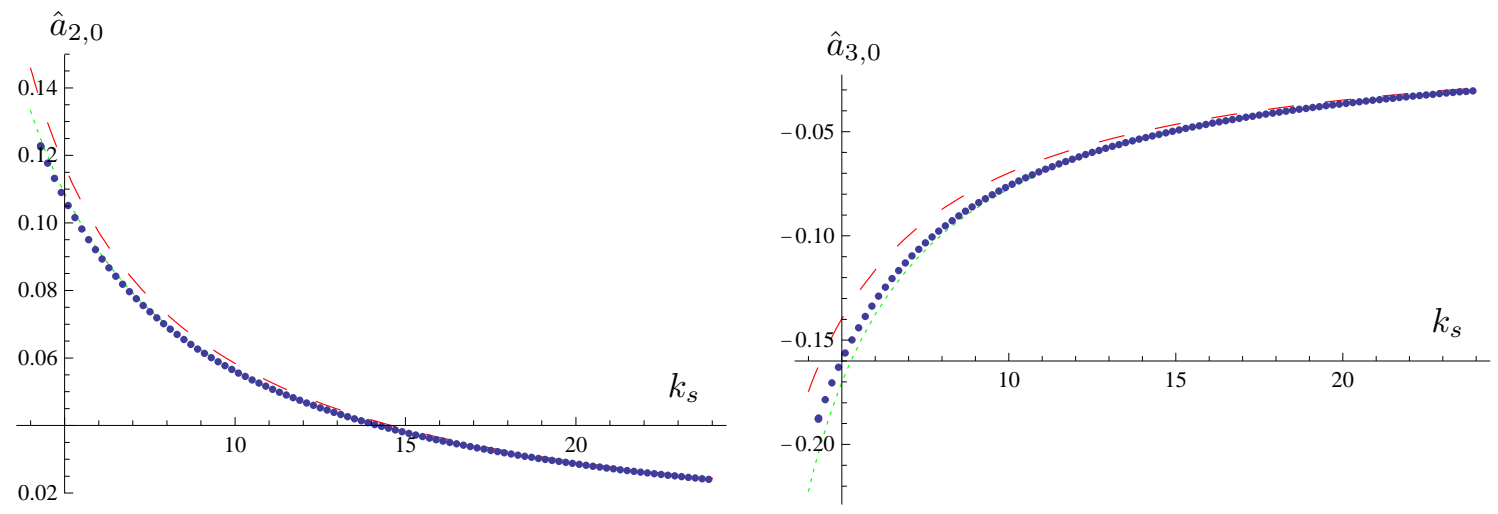

Figure 2: Values of the UV parameters $\hat{a}_{2,0}$ and $\hat{a}_{3,0}$ as a function of $k_{s}$ (blue points). The dashed/dotted (red/green) curves represent the perturbative $\mathcal{O}\left(k_{s}^{-1}\right) / \mathcal{O}\left(k_{s}^{-2}\right)$ asymptotics of the parameters, given by (5.5).
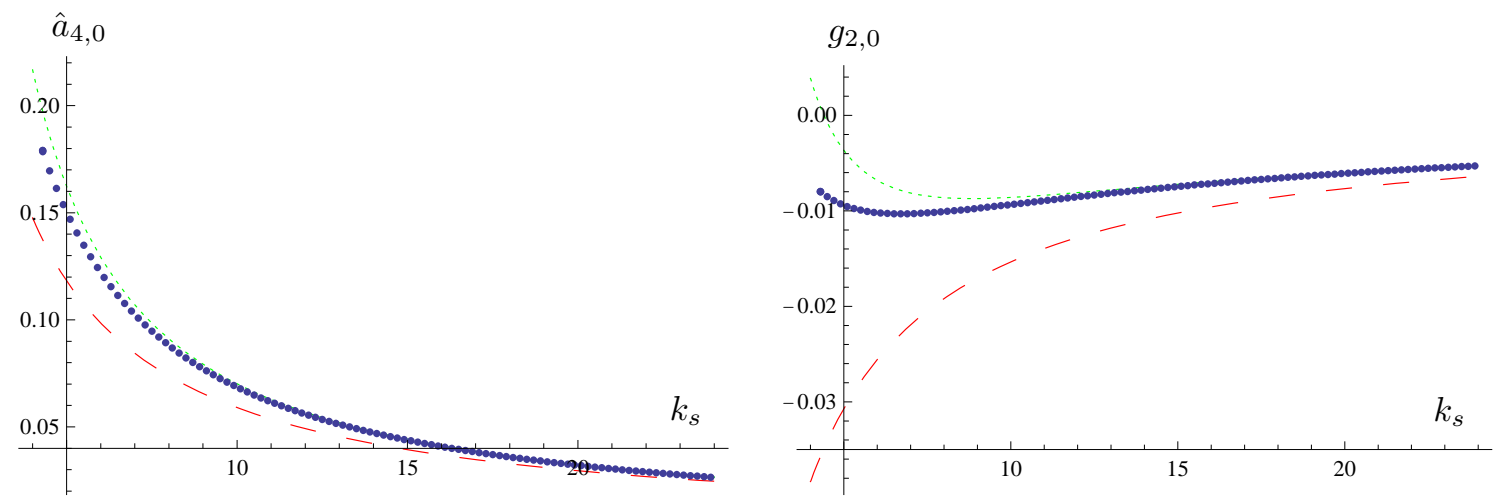

Figure 3: Values of the UV parameters $\hat{a}_{4,0}$ and $g_{2,0}$ as a function of $k_{s}$ (blue points). The dashed/dotted (red/green) curves represent the perturbative $\mathcal{O}\left(k_{s}^{-1}\right) / \mathcal{O}\left(k_{s}^{-2}\right)$ asymptotics of the parameters, given by (5.5).

- for large values of $k_{s}$, where we can check our numerical results against the perturbative analytic predictions (5.5);

- for an interval of small values of $k_{s}$ that includes the first order transition point to a confined thermal cascading background with broken chiral symmetry (as we will discuss in the next section).

\subsubsection{Large values of $k_{s}$}

Figures 2 and 3 present the dependence of the UV parameters $\left\{\hat{a}_{2,0}, \hat{a}_{3,0}, \hat{a}_{4,0}, g_{2,0}\right\}$ on $k_{s} \in(4.29,24.0)$, with a step of $\Delta k_{s}=0.01$ (blue points). In this regime the typical 

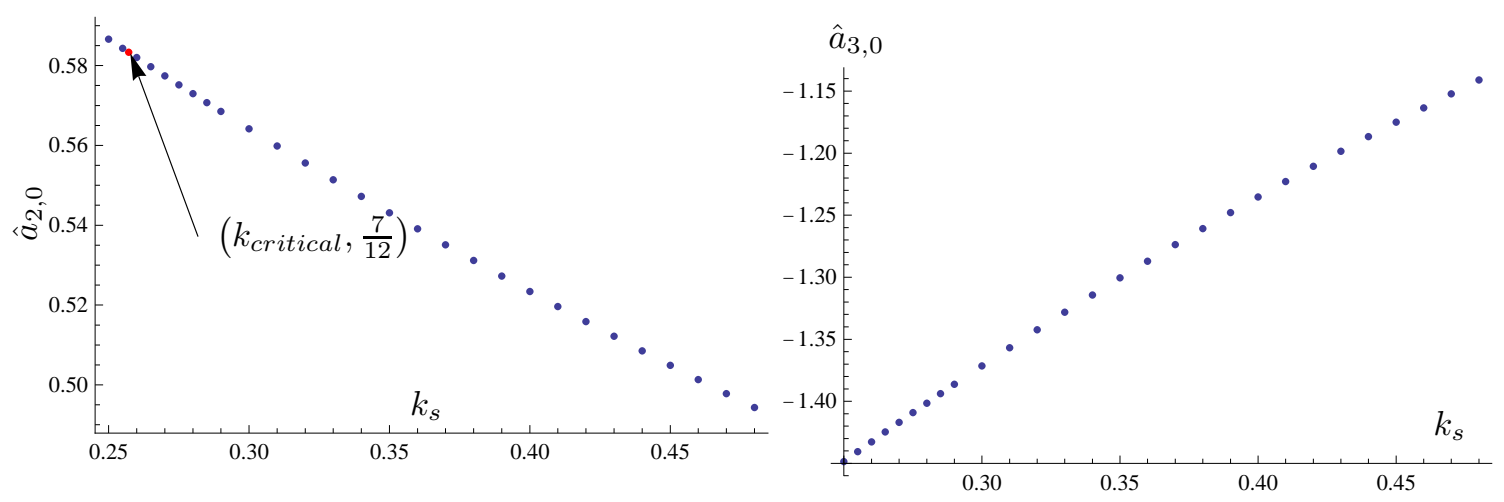

Figure 4: The values of the UV parameters $\hat{a}_{2,0}$ and $\hat{a}_{3,0}$ as a function of $k_{s}$.
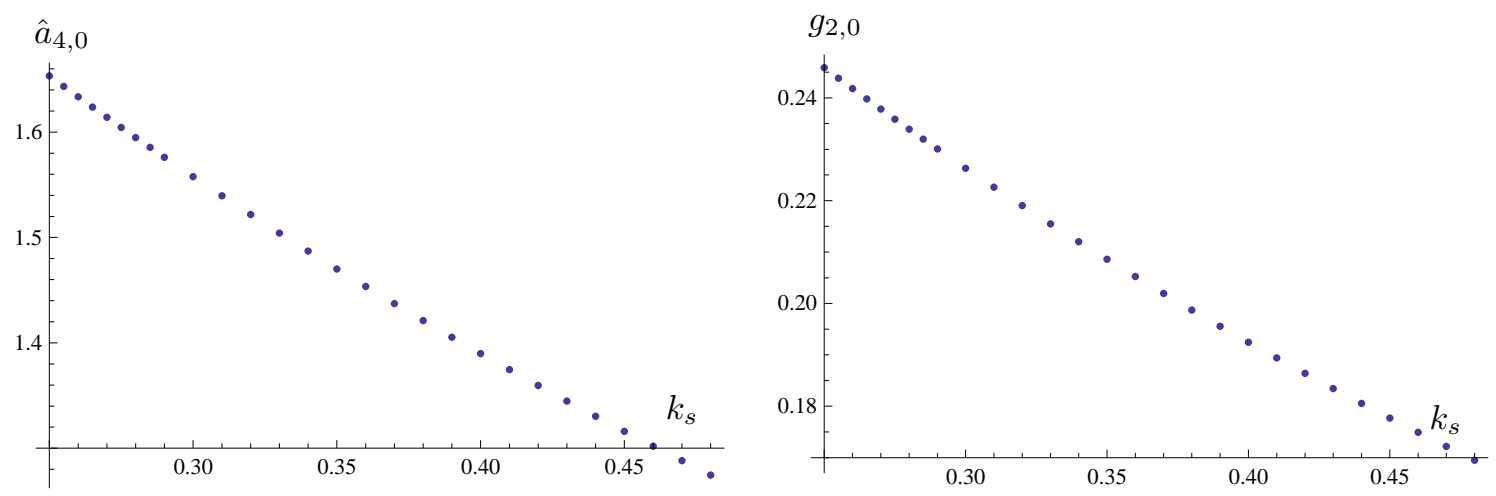

Figure 5: The values of the UV parameters $\hat{a}_{4,0}$ and $g_{2,0}$ as a function of $k_{s}$.

norm of the mismatch vector $(5.9)\left\|\vec{v}_{\text {mismatch }}\right\| \sim 10^{-10}$ or less. The dashed and dotted (red and green) curves represent the perturbative $\mathcal{O}\left(k_{s}^{-1}\right)$ and $\mathcal{O}\left(k_{s}^{-2}\right)$ asymptotics (5.5), respectively. Note that the dotted (green) curves track our numerical data quite well in this regime ${ }^{11}$.

\subsubsection{Small values of $k_{s}$}

Figures 4-8 present the dependence of the UV $\left\{\hat{a}_{2,0}, \hat{a}_{3,0}, \hat{a}_{4,0}, g_{2,0}\right\}$ and the IR $\left\{a_{0}^{h}, a_{1}^{h}\right.$, $\left.b_{0}^{h}, g_{0}^{h}, k_{0}^{h}, h_{0}^{h}\right\}$ parameters on $k_{s} \in(0.25,0.48)$ with a step of $\Delta k_{s}=0.01\left(\Delta k_{s}=0.005\right.$ near the transition) (blue points). In this regime the typical norm of the mismatch vector (5.9) $\left\|\vec{v}_{\text {mismatch }}\right\| \sim 10^{-5}$ or less. A highly non-trivial check on our numerics is the consistency of the holographic flow invariant $s T$. The latter can be computed in

\footnotetext{
${ }^{11}$ In appendix A we confirm using the perturbative high-temperature expansion that the cascading geometry thermodynamics satisfies the first law of thermodynamics up to order $\mathcal{O}\left(k_{s}^{-3}\right)$.
} 

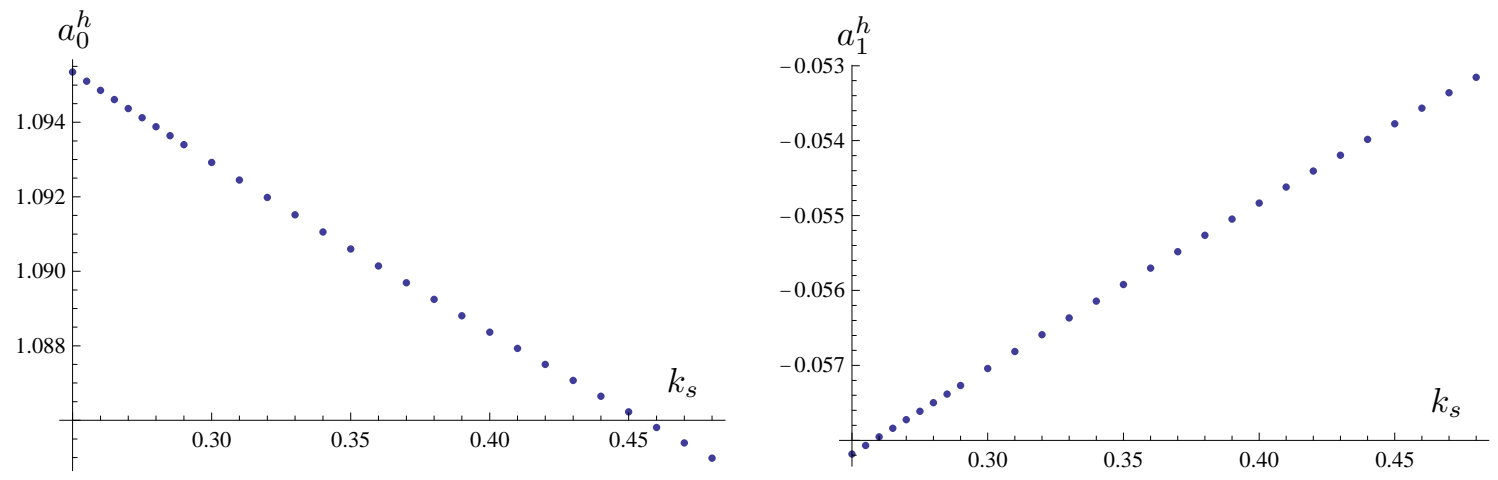

Figure 6: The values of the IR parameters $a_{0}^{h}$ and $a_{1}^{h}$ as a function of $k_{s}$.
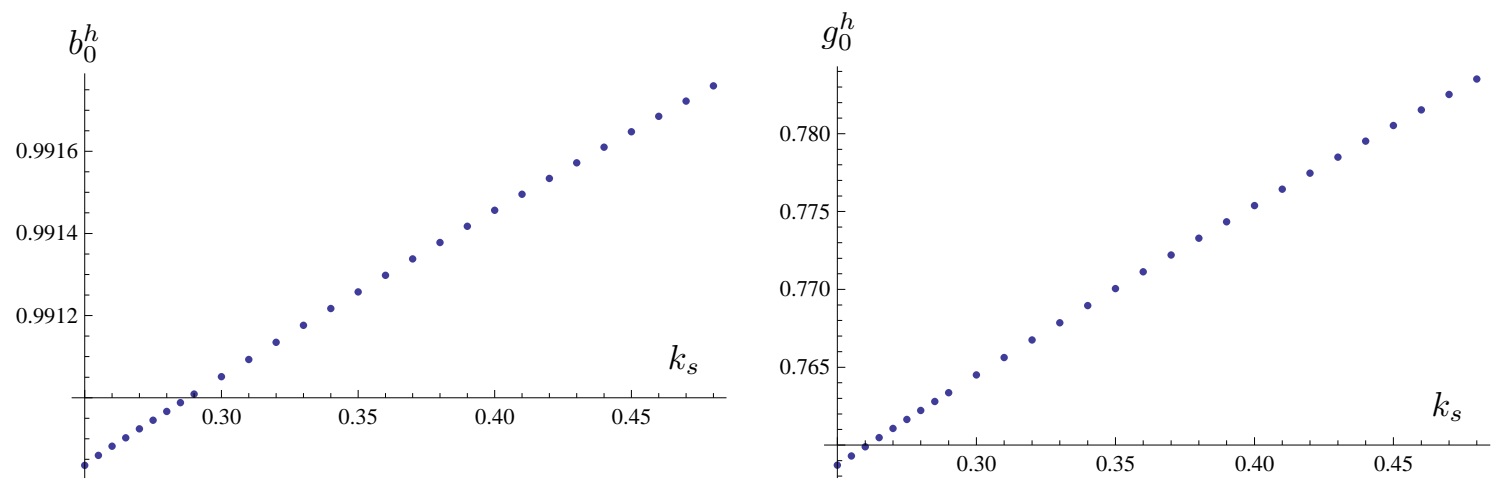

Figure 7: The values of the IR parameters $b_{0}^{h}$ and $g_{0}^{h}$ as a function of $k_{s}$.

the IR using (4.14), or in the UV using (4.13). We find that

$$
\left|\frac{\left.s T\right|_{I R}}{\left.s T\right|_{U V}}-1\right| \sim 10^{-5}
$$

or less, which provides an independent check on the accuracy of matching the IR and UV solutions (5.9).

The special (red) point in figure 4 denotes a critical value of $k_{s}=k_{\text {critical }}$, for which the corresponding value of the parameter $\hat{a}_{2,0}=\hat{a}_{2,0}\left(k_{\text {critical }}\right)=\frac{7}{12}$ leads to a vanishing of the free energy density $(4.11)$. We find $k_{\text {critical }}$ by performing a linear fit of the first 5 numerical points:

$$
k_{\text {critical }}=0.25712(1) .
$$

Our available numerical data shows that the free energy density (4.11)

$$
\mathcal{F}=\frac{3 a_{0}^{2}}{28 \pi G_{5}}\left(\hat{a}_{2,0}-\frac{7}{12}\right)
$$



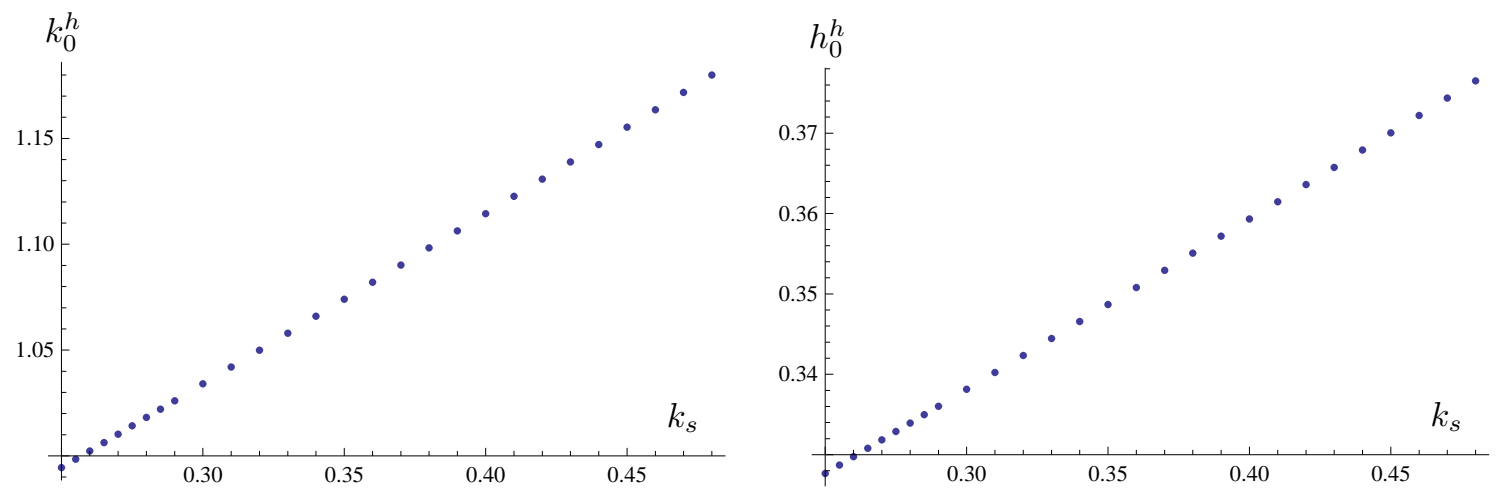

Figure 8: The values of the IR parameters $k_{0}^{h}$ and $h_{0}^{h}$ as a function of $k_{s}$.

is negative when $k_{s}>k_{\text {critical }}$ and is positive when $k_{s}<k_{\text {critical }}$, so we find that at temperatures lower than $k_{s}=k_{\text {critical }}$ the black hole solutions are not thermodynamically preferred over the gas of particles in the background of [9]. Thus, if we assume that these are the only two possible configurations, $k_{\text {critical }}$ gives a critical temperature corresponding to a first order confinement transition, with chiral symmetry breaking, in the gravitational dual to the cascading gauge theory. Examination of the infrared parameters in figures 6-8 shows that the geometry at this transition is non-singular (as expected for a first order transition), and can be made arbitrarily weakly curved for large values of $P$, justifying the validity of the supergravity approximation. This observation is the main result of our paper.

\section{The physical results obtained from our numerical solutions}

In this final section we translate the results of the previous section into physical quantities in the theory as a function of the temperature. We present all the results as a function of $T / \Lambda$, where the temperature $T$ is given by (4.14), and the scale $\Lambda$ enters through the temperature dependence of $k_{s}$. Recall that the numerical results presented in section 5 were obtained when setting $P=g_{0}=1$ and $a_{0}=1$. It is easy to restore the correct powers of $P$ using the scaling symmetry (5.4), and to then put a factor of $g_{0}=\hat{g}_{0}$ together with every factor of $P^{2}$. In order to relax the $a_{0}=1$ condition, all the dimensionful quantities must be computed in units of (see (5.2))

$$
\Lambda=e^{-k_{s} / 2}
$$


In particular, from (4.14) we have

$$
\frac{T}{\Lambda}=\frac{e^{k_{s} / 2}}{4 \pi h_{0}^{h} b_{0}^{h}} \sqrt{\frac{2\left(8 h_{0}^{h}\left(a_{0}^{h}\right)^{2}-g_{0}^{h}\right)}{a_{0}^{h}+2 a_{1}^{h}}},
$$

enabling us to translate the dependence on $k_{s}$ into a dependence on the temperature.

Equations (4.10) and (4.11) imply that the simplest expressions arise for the free energy density and the energy density divided by $s T$, which are given by

$$
\frac{\mathcal{F}}{s T}=\frac{3}{7}\left(\hat{a}_{2,0}-\frac{7}{12}\right), \quad \frac{\mathcal{E}}{s T}=\frac{3}{4}\left(1+\frac{4}{7} \hat{a}_{2,0}\right) .
$$

Equations (5.2) and (4.14) allow us to compute the entropy density divided by the temperature cubed, which is a measure of the number of degrees of freedom in the theory :

$$
\frac{4 \pi G_{5}}{P^{4} \hat{g}_{0}^{2}} \frac{s}{T^{3}}=\frac{32 \pi^{4} s T}{81 M^{4} \hat{g}_{0}^{2} T^{4}}=\frac{32 \pi^{4} s T}{81 M^{4} \hat{g}_{0}^{2} \Lambda^{4}}\left(\frac{\Lambda}{T}\right)^{4}=\left(\frac{1}{4 \pi h_{0}^{h} b_{0}^{h}} \sqrt{\frac{2\left(8 h_{0}^{h}\left(a_{0}^{h}\right)^{2}-g_{0}^{h}\right)}{a_{0}^{h}+2 a_{1}^{h}}}\right)^{-4}
$$

Notice that at high temperatures we can use the perturbative expression (A.42) of Appendix A and (4.13) to determine

$$
\frac{s}{T^{3}}=\frac{s T}{T^{4}}=\frac{a_{0}^{2}}{4 \pi G_{5} T^{4}}=\frac{\pi^{4} K_{\star}^{2}}{64 \pi G_{5}}\left(1+\mathcal{O}\left(\frac{P^{2} \hat{g}_{0}}{K_{\star}}\right)\right) \simeq \frac{81}{128} M^{4} \hat{g}_{0}^{2} \ln ^{2}\left(\frac{T}{\Lambda}\right) .
$$

Finally, we can evaluate the vacuum expectation values of the two dimension 4 scalar operators $(4.12)$ :

$$
\begin{aligned}
\frac{\left\langle\mathcal{O}_{K_{0}}\right\rangle}{\Lambda^{4}} & =\frac{24}{7} \frac{e^{2 k_{s}}}{P^{2} \hat{g}_{0}} \hat{a}_{2,0}, \\
\frac{\left\langle\mathcal{O}_{p_{0}}\right\rangle}{\Lambda^{4}} & =e^{2 k_{s}}\left(2 \frac{g_{2,0}}{\hat{g}_{0}^{2}}+\frac{12\left(1-\ln (2)+2 k_{s}\right)}{7 \hat{g}_{0}} \hat{a}_{2,0}\right) .
\end{aligned}
$$

Figure 9 presents $\ln \left(\frac{T}{\Lambda}\right)$ as a function of $k_{s}$ at low and high temperatures. This is useful to determine the temperature dependence of the various UV and IR parameters presented in figures 2-8. Notice that the high temperature dependence of $k_{s}$ is in a good agreement with the high temperature asymptotic analysis of appendix A. Indeed, a straight line fit of the points in the plot on the right determines the slope to be $0.46(3)$, while the $k_{s} \rightarrow \infty$ slope is expected to be $\frac{1}{2}$ (A.40). 


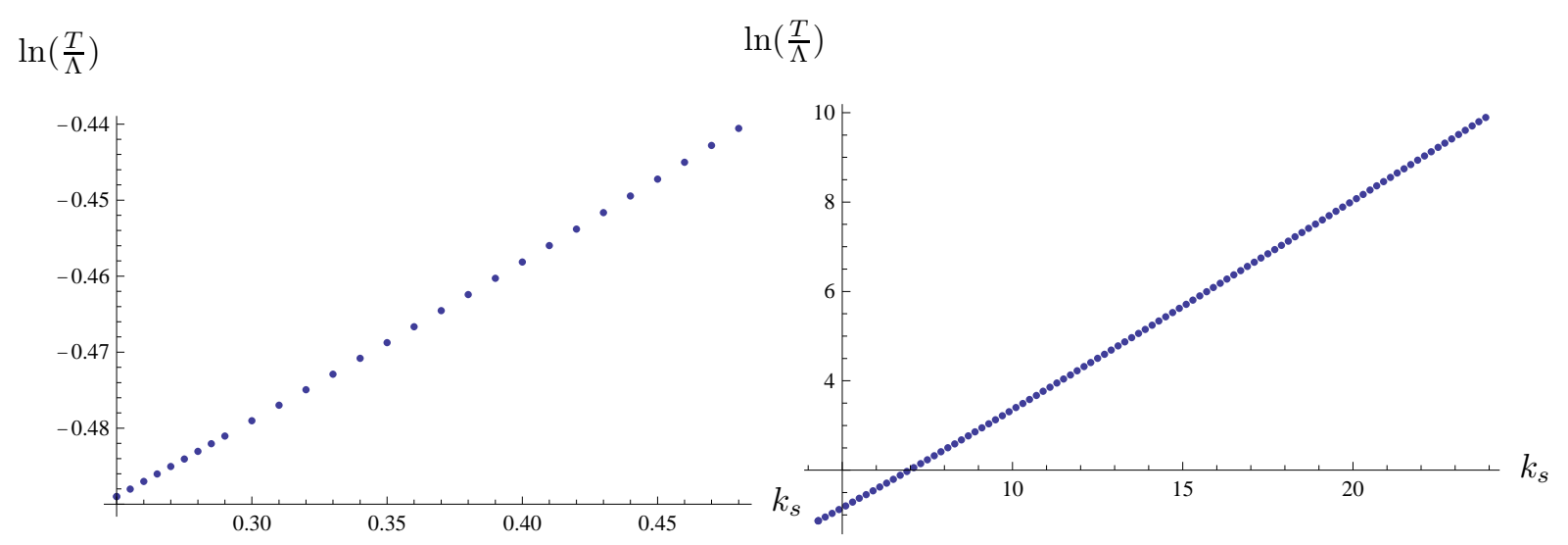

Figure 9: The relation between $k_{s}$ and the temperature $T$.
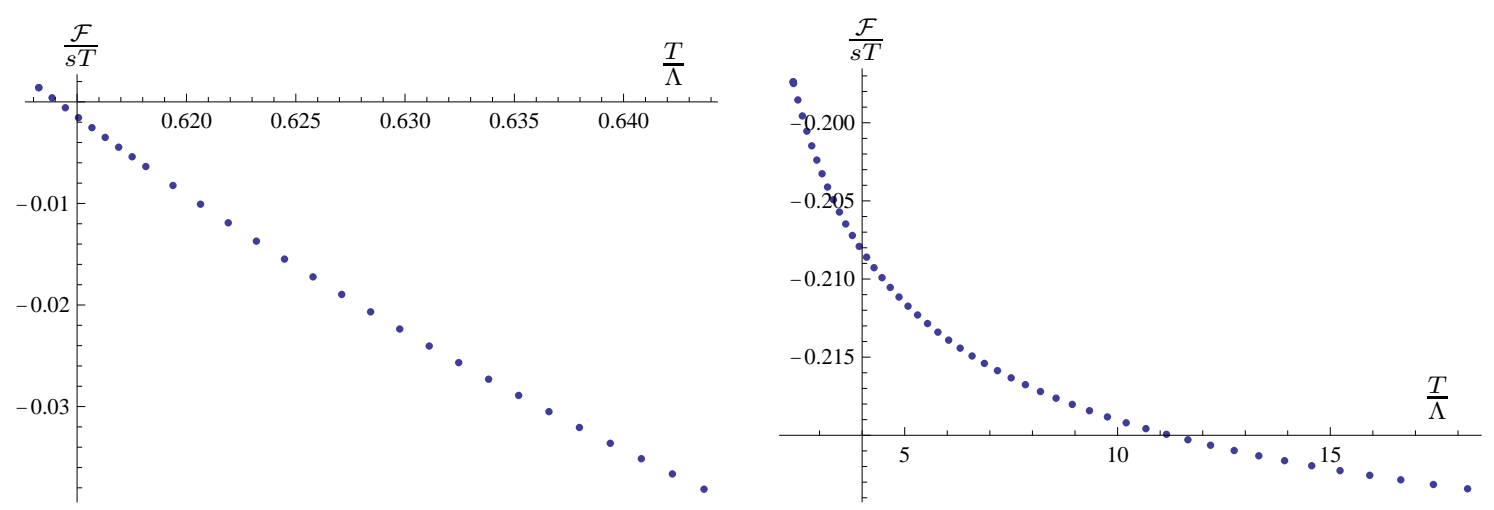

Figure 10: The free energy density $\mathcal{F}$, divided by $s T$, as a function of $\frac{T}{\Lambda}$. On the left we plot temperatures at and slightly above the deconfinement transition, and on the right much higher temperatures.

Figure 10 presents $\frac{\mathcal{F}}{s T}$ as a function of $\frac{T}{\Lambda}$ at low and high temperatures. Using a straight line fit of the first 6 points in the (left) free energy density plot, we determine the deconfinement and chiral symmetry restoration temperature to be

$$
\left(\frac{T}{\Lambda}\right)_{\text {critical }}=0.614111(3)
$$

by requiring that the free energy density vanishes at $T=T_{\text {critical }}$. Notice that there are noticeably large deviations from scale invariant thermodynamics even for rather large temperatures. Indeed, for $\frac{T}{\Lambda} \sim 10$, the deviation of $\frac{\mathcal{F}}{s T}$ from the conformal result

$$
\left.\frac{\mathcal{F}}{s T}\right|_{\text {conformal }}=-\frac{1}{4}
$$

is about $12 \%$. 

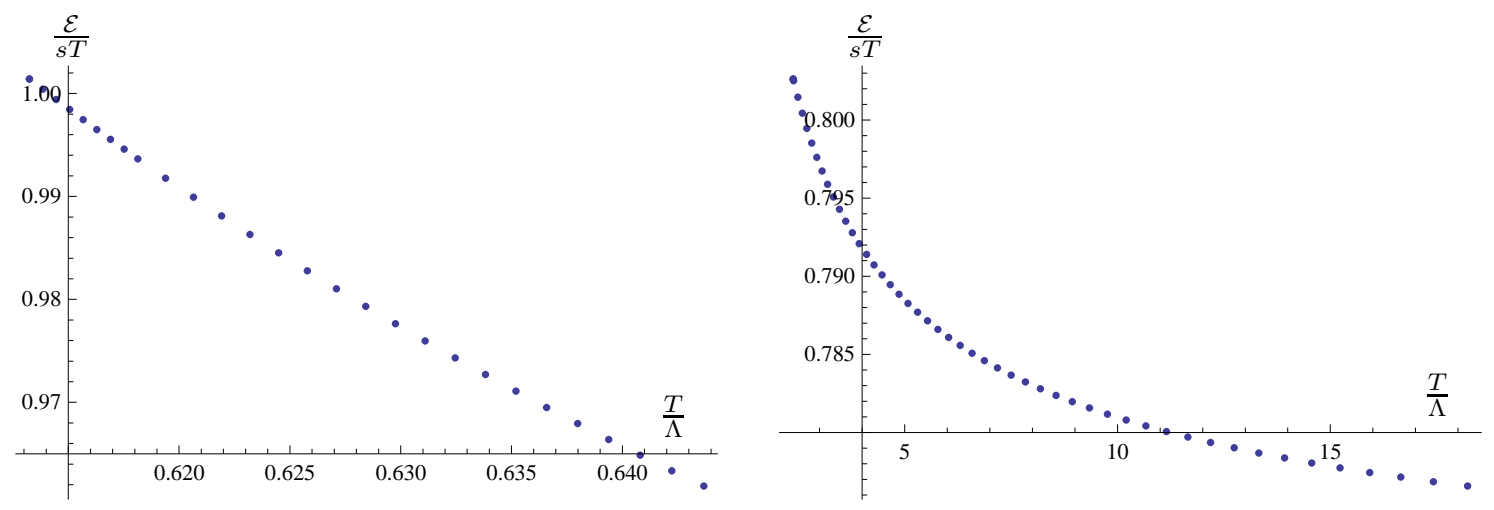

Figure 11: The energy density $\mathcal{E}$, divided by $s T$, as a function of $\frac{T}{\Lambda}$.
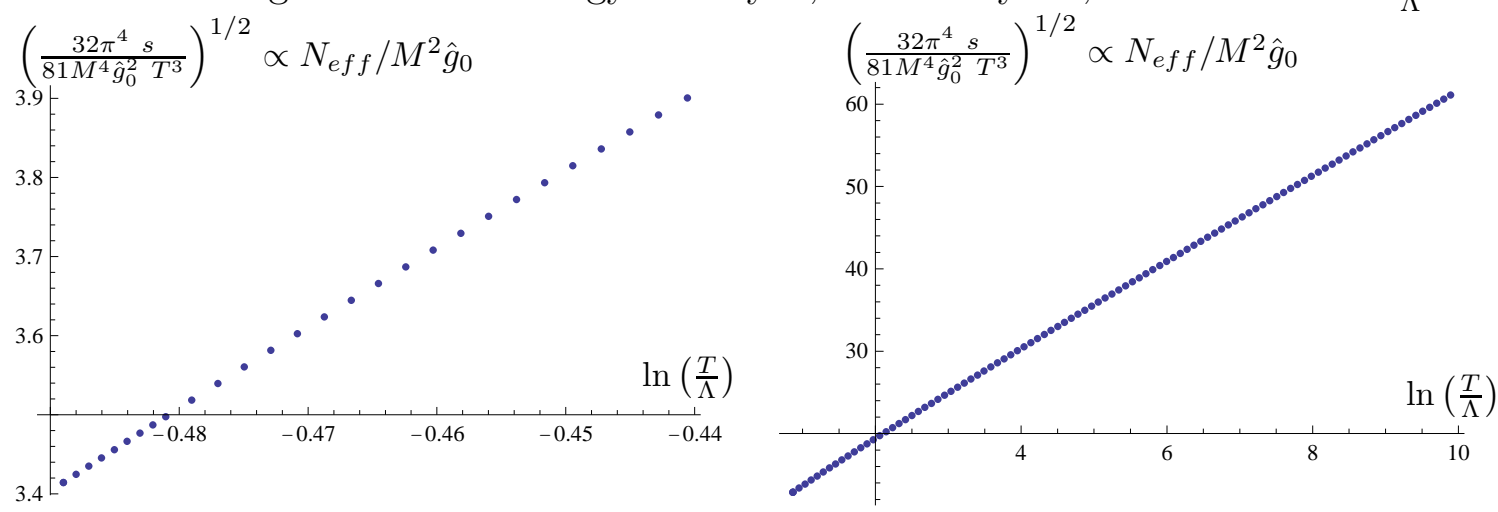

Figure 12: The temperature dependence of the effective number of degrees of freedom in the strongly coupled cascading gauge theory, as defined by $N_{e f f}^{2} \propto s / T^{3}$.

Figure 11 presents $\frac{\mathcal{E}}{s T}$ as a function of $\frac{T}{\Lambda}$ at low and high temperatures. Here, the deviation at high temperature from the conformal result

$$
\left.\frac{\mathcal{E}}{s T}\right|_{\text {conformal }}=\frac{3}{4}
$$

is three times less than the corresponding deviation in the free energy density (or pressure). Such a suppression is easy to understand once we notice from (6.3) that

$$
\frac{\mathcal{F}}{s T}=(1-\delta) \times\left.\frac{\mathcal{F}}{s T}\right|_{\text {conformal }}, \quad \frac{\mathcal{E}}{s T}=\left(1+\frac{1}{3} \delta\right) \times\left.\frac{\mathcal{E}}{s T}\right|_{\text {conformal }},
$$

where $\delta \equiv \frac{12}{7} \hat{a}_{2,0}$. Note that the lattice results for QCD also imply that the energy density of the QCD plasma near the deconfinement transition is much more similar to that of scale-invariant thermodynamics than the QCD pressure [38].

Figure 12 presents the temperature dependence of the effective number of degrees of freedom of the strongly coupled cascading gauge theory at low and high temperatures, 

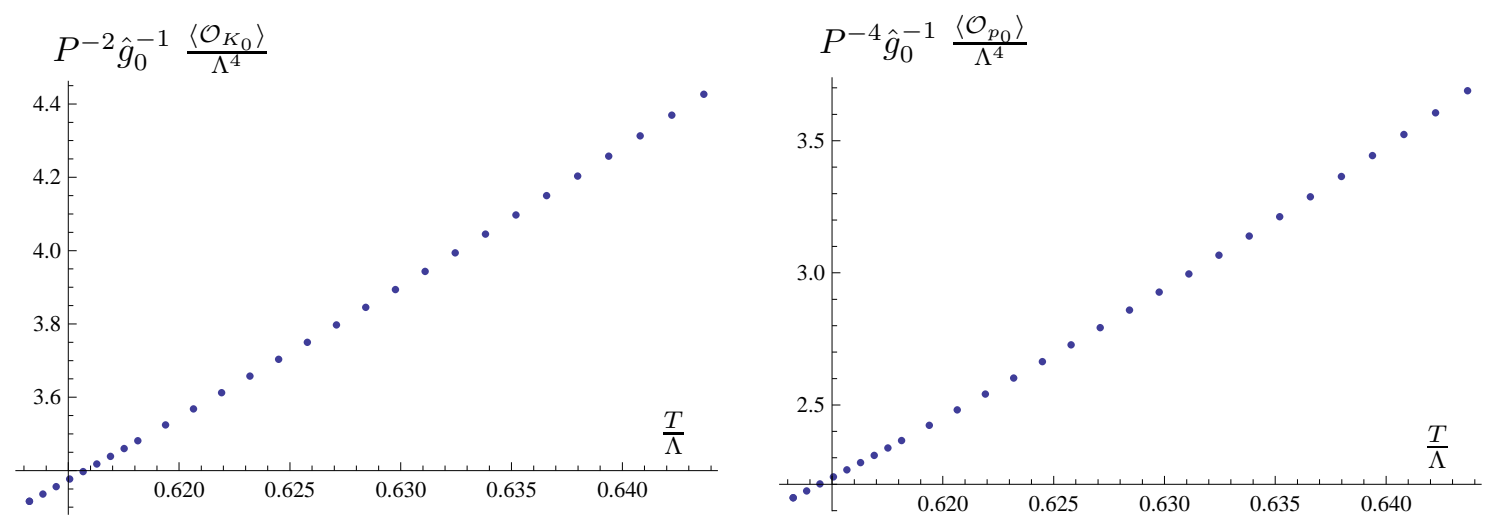

Figure 13: The temperature dependence of the vacuum expectation values of the dimension 4 operators $\left\langle\mathcal{O}_{K_{0}}\right\rangle$ and $\left\langle\mathcal{O}_{p_{0}}\right\rangle$. The operators are normalized such that they are invariant under the scaling transformation (5.4).

as defined by $N_{\text {eff }}^{2} \propto s / T^{3}$. One way to characterize the phase transition temperature is by the effective number of degrees of freedom (in the deconfined phase) at this temperature. Using the straight line fit of the first 6 points in the (left) effective number of degrees of freedom plot, we find that at the deconfinement and chiral symmetry restoration temperature $(6.7)$

$$
\left.\frac{32 \pi^{4}}{81 M^{4} \hat{g}_{0}^{2}} \frac{s}{T^{3}}\right|_{T=T_{\text {critical }}}=3.4291(5) .
$$

Figure 13 presents the temperature dependence of the vacuum expectation values of the dimension 4 operators $\left\langle\mathcal{O}_{K_{0}}\right\rangle$ and $\left\langle\mathcal{O}_{p_{0}}\right\rangle$ (see (6.6)) at low temperatures. At high temperatures we can use the perturbative expressions (A.42), (A.35) and (A.38) of appendix A to determine

$$
\begin{aligned}
P^{-2} \hat{g}_{0}^{-1} \frac{\left\langle\mathcal{O}_{K_{0}}\right\rangle}{\Lambda^{4}} & \propto\left(\frac{T}{\Lambda}\right)^{4} \ln \left(\frac{T}{\Lambda}\right) \\
P^{-4} \hat{g}_{0}^{-1} \frac{\left\langle\mathcal{O}_{p_{0}}\right\rangle}{\Lambda^{4}} & \propto\left(\frac{T}{\Lambda}\right)^{4} \ln ^{2}\left(\frac{T}{\Lambda}\right)
\end{aligned}
$$

\section{Acknowledgments}

We would like to thank Matthew Headrick, Igor Klebanov, Jim Liu, Chris Pagnutti, Leo Pando-Zayas, Larry Yaffe and Amos Yarom for interesting discussions. OA would like to thank the Institute for Advanced Study for hospitality during the course of this project. The work of OA is supported in part by the Israel-U.S. Binational Science 
Foundation, by a center of excellence supported by the Israel Science Foundation (grant number 1468/06), by the European network HPRN-CT-2000-00122, by a grant from the G.I.F., the German-Israeli Foundation for Scientific Research and Development, and by a grant of DIP (H.52). AB would like to thank the University of Texas at Austin for the hospitality, where part of this work was done. AB's research at Perimeter Institute is supported in part by the Government of Canada through NSERC and by the Province of Ontario through MEDT. AB gratefully acknowledges further support by an NSERC Discovery grant. PK would like to thank the Landesstiftung Baden-Wuerttemberg for the scholarship.

\section{A Appendix : Perturbative evaluation of the solutions at high temperature}

In this appendix we analyze the high temperature thermodynamics of the cascading gauge theory perturbatively in $P^{2} \hat{g}_{0} / K_{\star}$, where

$$
K_{\star}=P^{2} \hat{g}_{0}\left(k_{s}+\frac{1}{2} \ln (2)+\mathcal{O}\left(k_{s}^{-1}\right)\right)
$$

is the five-form flux evaluated at the horizon, and $k_{s}$ is defined by (5.1).

The purpose of this perturbative analysis is twofold: first, we would like to test our asymptotic identification of the cascading geometry parameters (4.5), of the temperature and of the dynamical scale $\Lambda$, against the first law of thermodynamics; second, we would like to obtain analytic predictions for the high-temperature values of the UV (5.7) and the IR parameters (5.8) perturbatively in $P^{2} \hat{g}_{0} / K_{\star}$, in order to benchmark our general numerical analysis. We will test the first law of thermodynamics to order $\mathcal{O}\left(\frac{P^{6} \hat{g}_{0}^{3}}{K_{\star}^{3}}\right)$ inclusive, and evaluate the UV parameters $(5.7)$ to order $\mathcal{O}\left(\frac{P^{4} \hat{g}_{0}^{2}}{K_{\star}^{2}}\right)$ inclusive.

The appendix is organized as follows. In subsection A.1 we derive the perturbative equations of motion to order $\mathcal{O}\left(\frac{P^{6} \hat{g}_{0}^{3}}{K_{\star}^{3}}\right)$ inclusive. In subsection A.2 we present the near horizon and the near boundary expansions of the solutions, outline our numerical method for the computation of the UV/IR parameters of the perturbative solutions, and collect numerical expressions for some of these parameters. In subsection A.3 we present perturbative expressions for the thermodynamics of the deconfined cascading gauge theory, and verify the first law of thermodynamics. 


\section{A.1 Perturbative equations of motion}

As discussed above, without loss of generality we can set $g_{0}=\hat{g}_{0}=1$. We use the following parametrization for the solution in perturbation theory in $\frac{P^{2}}{K_{\star}}$ :

$$
\begin{gathered}
h(x)=\frac{K_{\star}}{4 \tilde{a}_{0}^{2}}+\frac{K_{\star}}{\tilde{a}_{0}^{2}} \sum_{n=1}^{3}\left\{\left(\frac{P^{2}}{K_{\star}}\right)^{n}\left(\xi_{2 n}(x)-\frac{5}{4} \eta_{2 n}(x)\right)\right\}+\frac{K_{\star}}{\tilde{a}_{0}^{2}} \mathcal{O}\left(\frac{P^{8}}{K_{\star}^{4}}\right), \\
f_{2}(x)=\tilde{a}_{0}+\tilde{a}_{0} \sum_{n=1}^{3}\left\{\left(\frac{P^{2}}{K_{\star}}\right)^{n}\left(-2 \xi_{2 n}(x)+\eta_{2 n}(x)+\frac{4}{5} \lambda_{2 n}(x)\right)\right\}+\tilde{a}_{0} \mathcal{O}\left(\frac{P^{8}}{K_{\star}^{4}}\right), \\
f_{3}(x)=\tilde{a}_{0}+\tilde{a}_{0} \sum_{n=1}^{3}\left\{\left(\frac{P^{2}}{K_{\star}}\right)^{n}\left(-2 \xi_{2 n}(x)+\eta_{2 n}(x)-\frac{1}{5} \lambda_{2 n}(x)\right)\right\}+\tilde{a}_{0} \mathcal{O}\left(\frac{P^{8}}{K_{\star}^{4}}\right), \\
K(x)=K_{\star}+K_{\star} \sum_{n=1}^{3}\left\{\left(\frac{P^{2}}{K_{\star}}\right)^{n} \kappa_{2 n}(x)\right\}+K_{\star} \mathcal{O}\left(\frac{P^{8}}{K_{\star}^{4}}\right), \\
g(x)=1+\sum_{n=1}^{3}\left\{\left(\frac{P^{2}}{K_{\star}}\right)^{n} \zeta_{2 n}(x)\right\}+\mathcal{O}\left(\frac{P^{8}}{K_{\star}^{4}}\right) .
\end{gathered}
$$

The advantage of this parametrization is that the equations for $\left\{\xi_{2 n}, \eta_{2 n}, \lambda_{2 n}, \zeta_{2 n}\right\}$ decouple, once the (decoupled) equation for $\kappa_{2 n}$ is solved, at each order $(n=1,2,3)$ in perturbation theory. We find (for $n=1,2,3$ )

$$
\begin{gathered}
0=\kappa_{2 n}^{\prime \prime}+\frac{\kappa_{2 n}^{\prime}}{x-1}+\mathcal{J}_{\kappa}^{[2 n]} \\
0=\eta_{2 n}^{\prime \prime}+\frac{\eta_{2 n}^{\prime}}{x-1}-\frac{8 \eta_{2 n}}{x^{2}(x-2)^{2}}-\frac{2}{5} \kappa_{2}^{\prime} \kappa_{2 n}^{\prime}-\frac{8 \kappa_{2 n}}{3 x^{2}(x-2)^{2}}+\mathcal{J}_{\eta}^{[2 n]} \\
0=\xi_{2 n}^{\prime \prime}+\frac{\left(3 x^{2}-6 x+4\right) \xi_{2 n}^{\prime}}{x(x-1)(x-2)}-\frac{2}{3} \kappa_{2}^{\prime} \kappa_{2 n}^{\prime}+\mathcal{J}_{\xi}^{[2 n]} \\
0=\lambda_{2 n}^{\prime \prime}+\frac{\lambda_{2 n}^{\prime}}{x-1}-\frac{3 \lambda_{2 n}}{x^{2}(x-2)^{2}}-2 \kappa_{2}^{\prime} \kappa_{2 n}^{\prime}+\mathcal{J}_{\lambda}^{[2 n]}, \\
0=\zeta_{2 n}^{\prime \prime}+\frac{\zeta_{2 n}^{\prime}}{x-1}+2 \kappa_{2}^{\prime} \kappa_{2 n}^{\prime}+\mathcal{J}_{\zeta}^{[2 n]}
\end{gathered}
$$

where the source terms $\left\{\mathcal{J}_{\kappa}^{[2 n]}, \mathcal{J}_{\eta}^{[2 n]}, \mathcal{J}_{\xi}^{[2 n]}, \mathcal{J}_{\lambda}^{[2 n]}, \mathcal{J}_{\zeta}^{[2 n]}\right\}$ are functionals of the lower order solutions: $\kappa_{2 m}, \xi_{2 m}, \eta_{2 m}, \lambda_{2 m}, \zeta_{2 m}$, with $m<n$. Explicit expressions for the source term functionals are available from the authors upon request. 
The perturbative solutions to (A.7)-(A.11) must be regular at the horizon, and must have the appropriate KT asymptotics (3.1) near the boundary.

The leading order $(n=1)$ solution to (A.7)-(A.11) was obtained in [10]:

$$
\begin{gathered}
\kappa_{2}=-\frac{1}{2} \ln \left(2 x-x^{2}\right), \\
\eta_{2}=\frac{\left(x^{2}-2 x+2\right)}{20\left(2 x-x^{2}\right)}\left(\operatorname{dil} \log \left(2 \mathrm{x}-\mathrm{x}^{2}\right)-\frac{1}{6} \pi^{2}\right)-\frac{1}{15}+\frac{\ln \left(2 x-x^{2}\right)}{15}, \\
\xi_{2}=\frac{1}{12} \ln \left(2 x-x^{2}\right), \\
\zeta_{2}=\frac{\pi^{2}}{24}-\frac{1}{2} \operatorname{dilog}(1-\mathrm{x})+\frac{1}{2} \operatorname{dil} \log (2-\mathrm{x})-\frac{1}{2} \ln (\mathrm{x}) \ln (1-\mathrm{x}) .
\end{gathered}
$$

There is no simple expression for $\lambda_{2}$ - it is straightforward to write an appropriate solution using the Green's function for (A.10), but this explicit expression is not useful.

Similarly, although the higher order $n=2,3$ solutions to (A.7)-(A.11) could be presented in quadratures, these expressions are not useful. Rather, we identify the higher order solutions by specifying their asymptotic expressions near the horizon and near the boundary, along with the numerical values for the relevant integration constants.

\section{A.2 UV/IR asymptotics of the perturbative solutions}

The UV/IR parameters of the higher order perturbative solutions are found by solving the differential equations (A.7)-(A.11) numerically from the boundary $(x=0)$, and requiring the proper boundary conditions at the horizon, which are

$$
\lim _{x \rightarrow 1_{-}} \kappa_{2 n}^{\prime}=\lim _{x \rightarrow 1_{-}} \eta_{2 n}^{\prime}=\lim _{x \rightarrow 1_{-}} \xi_{2 n}^{\prime}=\lim _{x \rightarrow 1_{-}} \lambda_{2 n}^{\prime}=\lim _{x \rightarrow 1_{-}} \zeta_{2 n}^{\prime}=0, \quad n=2,3
$$

To begin, we present the asymptotics of $\lambda_{2}$. As $x \rightarrow 0_{+}$we find

$\lambda_{2}=\frac{2}{3} x+\lambda_{3}^{[2]} x^{3 / 2}+\frac{11}{15} x^{2}+\frac{3}{4} \lambda_{3}^{[2]} x^{5 / 2}+\frac{176}{315} x^{3}+\frac{9}{16} \lambda_{3}^{[2]} x^{7 / 2}+\frac{676}{1575} x^{4}+\frac{7}{16} \lambda_{3}^{[2]} x^{9 / 2}+\mathcal{O}\left(x^{5}\right)$,

where $\lambda_{3}^{[2]}$ is related to the condensate of the dimension 6 operator at order $\mathcal{O}\left(\frac{P^{2}}{K_{\star}}\right)$. Nonsingularity of the $\lambda_{2}(x)$ solution to (A.10) at the horizon, together with

$$
\lim _{x \rightarrow 1_{-}} \lambda_{2}^{\prime}=0
$$

determines

$$
\lambda_{3}^{[2]}=-0.872358024(9) .
$$


Near the horizon, as $y \equiv 1-x \rightarrow 0_{+}$, we find

$$
\begin{aligned}
\lambda_{2}= & \lambda_{2,0}^{h}+\left(-\frac{1}{4}+\frac{3}{4} \lambda_{2,0}^{h}\right) y^{2}+\left(\frac{33}{64} \lambda_{2,0}^{h}-\frac{7}{64}\right) y^{4}+\left(\frac{107}{256} \lambda_{2,0}^{h}-\frac{181}{2304}\right) y^{6} \\
& +\left(-\frac{3181}{49152}+\frac{5913}{16384} \lambda_{2,0}^{h}\right) y^{8}+\mathcal{O}\left(y^{10}\right)
\end{aligned}
$$

where $\lambda_{2,0}^{h}$ can be determined numerically to be

$$
\lambda_{2,0}^{h}=0.16806(9)
$$

\section{A.2.1 Order $n=2$ asymptotics}

- As $x \rightarrow 0_{+}$we find

$$
\begin{aligned}
\kappa_{4}= & \left(\kappa_{2}^{[4]}-\frac{1}{2} \ln (x)\right) x+\frac{2}{15} \lambda_{3}^{[2]} x^{3 / 2}+\left(-\frac{106}{225}+\frac{7}{30} \ln (2)+\frac{1}{2} \kappa_{2}^{[4]}-\frac{1}{60} \ln (x)\right) x^{2} \\
& +\frac{1}{50} \lambda_{3}^{[2]} x^{5 / 2}+\mathcal{O}\left(x^{3}\right) \\
\eta_{4}= & \left(-\frac{1}{12}+\frac{1}{18} \ln (2)+\frac{1}{18} \ln (x)\right)+\left(\frac{13}{360}-\frac{1}{30} \ln (2)-\frac{7}{30} \kappa_{2}^{[4]}+\frac{1}{12} \ln (x)\right) x \\
& -\frac{4}{225} \lambda_{3}^{[2]} x^{3 / 2}+\left(\eta_{4}^{[4]}+\left(\frac{7}{360}-\frac{1}{36} \ln (2)+\frac{1}{15} \kappa_{2}^{[4]}\right) \ln (x)-\frac{11}{360} \ln ^{2}(x)\right) x^{2} \\
& -\frac{97}{1575} \lambda_{3}^{[2]} x^{5 / 2}+\mathcal{O}\left(x^{3}\right), \\
\xi_{4}= & \frac{1}{36} \ln (x)+\left(-\frac{1}{6} \kappa_{2}^{[4]}+\frac{11}{144}-\frac{1}{24} \ln (2)+\frac{1}{24} \ln (x)\right) x-\frac{2}{225} \lambda_{3}^{[2]} x^{3 / 2} \\
+ & \left(-\frac{191}{43200}-\frac{1}{36} \kappa_{2}^{[4]}+\frac{1}{80} \ln (2)-\frac{1}{144} \ln ^{2}(2)+\left(\frac{19}{720}-\frac{1}{72} \ln (2)\right) \ln (x)\right. \\
+ & \left.\left.\frac{1}{144} \ln ^{2}(x)\right) x^{2}-\frac{229}{3150} \lambda_{3}^{[2]} x^{5 / 2}+\mathcal{O}\left(x^{3}\right), 223\right) \\
\lambda_{4}= & \left(-\frac{14}{9}+\frac{4}{3} \kappa_{2}^{[4]}+\frac{2}{3} \ln (2)\right) x+\lambda_{3}^{[4]} x^{3 / 2}+\left(\frac{67}{450}+\frac{22}{15} \kappa_{2}^{[4]}+\frac{1}{3} \ln (2)-\frac{2}{5} \ln x\right) x^{2} \\
+ & \left(\frac{4}{5} \lambda_{3}^{[2]}+\frac{3}{4} \lambda_{3}^{[4]}\right) x^{5 / 2}+\mathcal{O}\left(x^{3}\right),
\end{aligned}
$$




$$
\begin{aligned}
\zeta_{4}= & \left(\zeta_{2}^{[4]}+\left(-\frac{13}{12}+\kappa_{2}^{[4]}+\frac{1}{2} \ln (2)\right) \ln (x)\right) x \\
& +\left(-\frac{13}{24}+\frac{1}{2} \zeta_{2}^{[4]}+\frac{1}{8} \ln ^{2}(2)+\left(-\frac{13}{24}+\frac{1}{2} \kappa_{2}^{[4]}+\frac{1}{2} \ln (2)\right) \ln (x)+\frac{1}{8} \ln ^{2}(x)\right) x^{2} \\
& -\frac{4}{75} \lambda_{3}^{[2]} x^{5 / 2}+\mathcal{O}\left(x^{3}\right),
\end{aligned}
$$

where the new UV parameters $\left\{\kappa_{2}^{[4]}, \eta_{4}^{[4]}, \lambda_{3}^{[4]}, \zeta_{2}^{[4]}\right\}$ are determined numerically from the horizon boundary condition (A.16):

$$
\left.\kappa_{2}^{[4]}\right|_{\text {numeric }}=0.73675974(3),
$$

and

$$
\begin{aligned}
& \left.\eta_{4}^{[4]}\right|_{\text {numeric }}=0.0053421556(6), \\
& \left.\lambda_{3}^{[4]}\right|_{\text {numeric }}=-1.1156300100(2), \\
& \left.\zeta_{2}^{[4]}\right|_{\text {numeric }}=0.622262593(4) .
\end{aligned}
$$

With (A.26) and (A.27) there are no additional UV parameters to tune in order to enforce the horizon boundary condition (A.16) for $\xi_{4}$. We find

$$
\left.\xi_{4}^{\prime}(x=0.99999)\right|_{\text {numeric }} \sim 10^{-6},
$$

which is of the same order of magnitude as the error in (A.16) for all other functions. - The asymptotic expressions of the $n=2$ solutions near the horizon $y \equiv 1-x \rightarrow 0_{+}$ take the form

$$
\begin{aligned}
\kappa_{4} & =\kappa_{4,0}^{h}+\kappa_{4,2}^{h} y^{2}+\kappa_{4,4}^{h} y^{4}+\mathcal{O}\left(y^{6}\right), \\
\eta_{4} & =\eta_{4,0}^{h}+\eta_{4,2}^{h} y^{2}+\eta_{4,4}^{h} y^{4}+\mathcal{O}\left(y^{6}\right), \\
\xi_{4} & =\xi_{4,0}^{h}+\xi_{4,2}^{h} y^{2}+\xi_{4,4}^{h} y^{4}+\mathcal{O}\left(y^{6}\right), \\
\lambda_{4} & =\lambda_{4,0}^{h}+\lambda_{4,2}^{h} y^{2}+\lambda_{4,4}^{h} y^{4}+\mathcal{O}\left(y^{6}\right), \\
\zeta_{4} & =\zeta_{4,0}^{h}+\zeta_{4,2}^{h} y^{2}+\zeta_{4,4}^{h} y^{4}+\mathcal{O}\left(y^{6}\right) .
\end{aligned}
$$

To verify the first law of thermodynamics to order $\mathcal{O}\left(\frac{P^{6}}{K \star^{3}}\right)$ we will need the numerical expressions only for $\left\{\kappa_{4,0}^{h}, \xi_{4,0}^{h}, \xi_{4,2}^{h}\right\}$. We find

$$
\left.\kappa_{4,0}^{h}\right|_{\text {numeric }}=0.62226(3),
$$


and

$$
\begin{aligned}
& \left.\xi_{4,0}^{h}\right|_{\text {numeric }}=-0.079819(3), \\
& \left.\xi_{4,2}^{h}\right|_{\text {numeric }}=0.019198(8) .
\end{aligned}
$$

\section{A.2.2 Order $n=3$ asymptotics}

To verify the first law of thermodynamics to order $\mathcal{O}\left(\frac{P^{6}}{K \star^{3}}\right)$ we will need the asymptotic expression for $\kappa_{6}$ only. We find

$$
\begin{aligned}
\kappa_{6}= & \left(\kappa_{2}^{[6]}+\left(\frac{13}{12}-\kappa_{2}^{[4]}-\frac{1}{2} \ln (2)\right) \ln (x)\right) x+\left(\frac{1}{45} \lambda_{3}^{[2]}+\frac{2}{15} \lambda_{3}^{[4]}\right) x^{3 / 2} \\
& +\left(\frac{281821}{216000}-\frac{2587}{3600} \kappa_{2}^{[4]}-\frac{509}{720} \ln (2)+\frac{31}{288} \ln ^{2}(2)+\frac{1}{2} \kappa_{2}^{[6]}+\frac{13}{4} \eta_{4}^{[4]}+\frac{1}{4} \zeta_{2}^{[4]}\right. \\
& \left.+\frac{1}{4} \ln (2) \kappa_{2}^{[4]}+\left(-\frac{49}{3600}+\frac{13}{60} \kappa_{2}^{[4]}-\frac{1}{8} \ln (2)\right) \ln (x)-\frac{7}{60} \ln ^{2}(x)\right) x^{2} \\
& +\left(\frac{1153}{10500} \lambda_{3}^{[2]}-\frac{4}{25} \lambda_{3}^{[2]} \kappa_{2}^{[4]}+\frac{1}{50} \lambda_{3}^{[4]}+\frac{2}{25} \lambda_{3}^{[2]} \ln (x)\right) x^{5 / 2}+\mathcal{O}\left(x^{3}\right)
\end{aligned}
$$

as $x \rightarrow 0_{+}$, with

$$
\kappa_{2}^{[6]}=-0.62226259(3)
$$

determined from the horizon boundary condition (A.16).

\section{A.2.3 Perturbative expressions for UV parameters (3.1)}

Finally, we collect perturbative expressions for the various independent UV parameters $a_{2,0}, a_{3,0}, a_{4,0}, g_{2,0}$ as defined by (3.1). Because of the scaling symmetry (4.1) it is convenient to quote these parameters relative to $a_{0}$. Also, in the next subsection we show that the first law of thermodynamics requires that

$$
\kappa_{2}^{[4]}=\frac{13}{12}-\frac{1}{2} \ln (2) .
$$

Note that (A.34) agrees with (A.26) up to an error of order $10^{-10}$. In the following expressions for the UV parameters in (3.1) we use the analytic expression (A.34).

We find

$$
\frac{a_{2,0}}{a_{0}}=\frac{7}{12} \frac{P^{2}}{K_{\star}}+\mathcal{O}\left(\frac{P^{6}}{K_{\star}^{3}}\right),
$$




$$
\begin{gathered}
\frac{a_{3,0}}{a_{0}}=\frac{4}{5} \lambda_{3}^{[2]} \frac{P^{2}}{K_{\star}}+\left(\frac{4}{5} \lambda_{3}^{[4]}+\frac{2}{15} \lambda_{3}^{[2]}\right) \frac{P^{4}}{K_{\star}^{2}}+\mathcal{O}\left(\frac{P^{6}}{K_{\star}^{3}}\right) \\
\frac{a_{4,0}}{a_{0}}=\left(\frac{1}{30} \ln (2)+\frac{1021}{1800}\right) \frac{P^{2}}{K_{\star}}+\left(\eta_{4}^{[4]}-\frac{661}{1800} \ln (2)+\frac{1}{72}(\ln (2))^{2}+\frac{167809}{108000}\right) \frac{P^{4}}{K_{\star}^{2}} \\
+\mathcal{O}\left(\frac{P^{6}}{K_{\star}^{3}}\right) \\
g_{2,0}=\left(-\frac{1}{2}+\frac{1}{2} \ln (2)\right) \frac{P^{2}}{K_{\star}}+\zeta_{2}^{[4]} \frac{P^{4}}{K_{\star}^{2}}+\mathcal{O}\left(\frac{P^{6}}{K_{\star}^{3}}\right)
\end{gathered}
$$

\section{A.3 Perturbative thermodynamics of the non-extremal cascading geome- try}

One of the interesting properties of the deconfined cascading geometry is the temperature dependence of the five-form flux evaluated at the horizon $K_{\star}=K_{\star}(T)$. The need for such dependence was first pointed out in [10]; it stems from the fact that when studying the thermodynamics of non-conformal gauge theories (such as the cascading gauge theory) one must keep the intrinsic scale of the cascading gauge theory fixed ${ }^{12}$, rather than keeping fixed the five-form at the horizon.

The fact that $K_{\star}$ is temperature dependent introduces additional temperature dependence into the thermodynamic potentials (the free energy density $\mathcal{F}(4.11)$, the energy density $\mathcal{E}(4.10)$, and the entropy density $s(4.14))$ via the UV parameters $a_{0}$ and $a_{2,0}$, both of which depend on $K_{\star}$. As a result, the first law of thermodynamics

$$
d \mathcal{F}=-s d T
$$

would not be valid, unless the temperature dependence of $K_{\star}$ is properly determined and taken into account. One possible approach is to use the first law of thermodynamics (A.39) as a way to determine $K_{\star}(T)$. Such an approach was proposed and implemented in [30] to leading order in $\mathcal{O}\left(\frac{P^{2}}{K_{\star}}\right)$, where it was found that validity of (A.39) requires that

$$
\frac{d K_{\star}(T)}{d T}=\frac{2 P^{2}}{T}+\mathcal{O}\left(\frac{P^{4}}{K_{\star}}\right)
$$

(this was also proposed in [10], based on the requirement of keeping the glueball spectrum scale of the cascading gauge theory fixed). Equation (A.40) was also shown to

\footnotetext{
${ }^{12}$ This fact was not clearly taken into account in previous numerical studies [13].
} 
be required for the consistency of the hydrodynamics of the cascading gauge theory plasma in [18].

The main observation of this paper is that one can rigorously determine the temperature dependence of $K_{\star}$ without referring to the first law of thermodynamics. In the bulk of the paper this was implicitly done in our solutions. In the context of the perturbative high-temperature expansion, we can obtain such an identification perturbatively by expanding the exact matching condition (4.5), enforcing the fixed scale of the cascading gauge theory, perturbatively in $\frac{P^{2}}{K_{\star}}$. We will demonstrate here that this identification is consistent with the first law of thermodynamics. This provides a non-trivial consistency check on our solutions.

In the rest of this subsection we present explicit expressions for $a_{0}$ as a function of the temperature $T$ to order $\mathcal{O}\left(\frac{P^{4}}{K_{\star}^{2}}\right)$. One can then use (A.35) to compute the thermodynamic potentials of the cascading black hole geometry. We present explicit perturbative expressions for $\frac{d K_{\star}(T)}{d T}$, and verify the first law of thermodynamics to order $\mathcal{O}\left(\frac{P^{6}}{K_{\star}^{3}}\right)$.

\section{A.3.1 Cascading black hole thermodynamics to order $\mathcal{O}\left(\frac{P^{2}}{K_{\star}}\right)$}

Explicitly evaluating the temperature of the black hole to order $\mathcal{O}\left(\frac{P^{2}}{K_{\star}}\right)$ we find

$$
\tilde{a}_{0}=\frac{\pi^{2} K_{\star} T^{2}}{4}\left(1+\frac{2 P^{2}}{3 K_{\star}}+\mathcal{O}\left(\frac{P^{4}}{K_{\star}^{2}}\right)\right) .
$$

Using (A.12)-(A.15), we further determine

$$
a_{0}=\frac{\pi^{2} K_{\star} T^{2}}{4}\left(1+\frac{P^{2}}{2 K_{\star}}+\mathcal{O}\left(\frac{P^{4}}{K_{\star}^{2}}\right)\right) .
$$

The matching condition (4.5) then determines

$$
\mathcal{O}\left(\frac{P^{4}}{K_{\star}}\right)=4 \hat{h}_{0,0}-K_{\star}+P^{2}\left(\frac{1}{2} \ln \left(\frac{\pi^{4} T^{4} K_{\star}^{2}}{16}\right)-\frac{1}{2}\right) .
$$

Assuming that $K_{\star}=K_{\star}(T)$ and differentiating (A.43), we find (A.40).

\section{A.3.2 Cascading black hole thermodynamics to order $\mathcal{O}\left(\frac{P^{4}}{K_{\star}^{2}}\right)$}

We can evaluate the black hole temperature to order $\mathcal{O}\left(\frac{P^{4}}{K_{\star}^{2}}\right)$ by requiring that the Euclidean continuation of the metric (2.4) does not have a conical singularity as $y \rightarrow 0_{+}$. 
We find ${ }^{13}$

$$
\begin{aligned}
T= & \frac{2}{\pi}\left(\frac{\tilde{a}_{0}}{K_{\star}}\right)^{1 / 2}\left\{1-\frac{P^{2}}{3 K_{\star}}+\frac{P^{4}}{K_{\star}^{2}}\left(\frac{\left(\pi^{2}+8\right)^{2}}{1920}+\frac{1}{30}\left(\lambda_{2,0}^{h}\right)^{2}-\xi_{4,0}^{h}+2 \xi_{4,2}^{h}-\frac{2}{3} \kappa_{4,0}^{h}\right)\right. \\
& \left.+\mathcal{O}\left(\frac{P^{6}}{K_{\star}^{3}}\right)\right\} .
\end{aligned}
$$

Solving for $\tilde{a}_{0}$ from (A.44), and reading off $\left\{a_{0}, a_{2,0}\right\}$ in (3.1) from (A.2)-(A.6), we find

$$
\begin{gathered}
a_{0}=\frac{1}{4} T^{2} \pi^{2} K_{\star}\left\{1+\frac{P^{2}}{2 K_{\star}}+\frac{P^{4}}{K_{\star}^{2}}\left(\frac{13}{180}+\frac{1}{18} \ln (2)-\frac{\pi^{4}}{960}-\frac{\pi^{2}}{60}-\frac{1}{15}\left(\lambda_{2,0}^{h}\right)^{2}+\frac{4}{3} \kappa_{4,0}^{h}\right.\right. \\
\left.\left.+2 \xi_{4,0}^{h}-4 \xi_{4,2}^{h}\right)+\mathcal{O}\left(\frac{P^{6}}{K_{\star}^{3}}\right)\right\} \\
a_{2,0}=\frac{7}{48} T^{2} \pi^{2} P^{2}\left\{1+\frac{P^{2}}{2 K_{\star}}+\mathcal{O}\left(\frac{P^{4}}{K_{\star}^{2}}\right)\right\}
\end{gathered}
$$

Additionally we find (see (5.1))

$$
P^{2} k_{s} \equiv 4 h_{0,0} a_{0}^{2}-\frac{1}{2} P^{2}=K_{\star}\left\{1-\frac{\ln (2)}{2} \frac{P^{2}}{K_{\star}}+\mathcal{O}\left(\frac{P^{6}}{K_{\star}^{3}}\right)\right\} .
$$

We are now ready to verify the first law of thermodynamics. The matching condition $(4.5)$, to order $\mathcal{O}\left(\frac{P^{4}}{K_{\star}^{2}}\right)$, gives

$$
\mathcal{O}\left(\frac{P^{6}}{K_{\star}^{2}}\right)=4 \hat{h}_{0,0}-K_{\star}+P^{2}\left(\frac{1}{2} \ln \left(\frac{\pi^{4} T^{4} K_{\star}^{2}}{16}\right)-\frac{1}{2}\right)+\frac{P^{4}}{2 K_{\star}},
$$

which results in the following ordinary differential equation for $K_{\star} \equiv K_{\star}(T)$ :

$$
\frac{d K_{\star}}{d T}=\frac{2 P^{2}}{T}\left\{1+\frac{P^{2}}{K_{\star}}+\mathcal{O}\left(\frac{P^{4}}{K_{\star}^{2}}\right)\right\} .
$$

Now, given (A.45) and (A.46) we can evaluate the energy density $\mathcal{E}$ and the pressure $\mathcal{P}$. The first law of thermodynamics (A.39) leads to

$$
\frac{d K_{\star}}{d T}=\frac{2 P^{2}}{T}\left\{1+\frac{P^{2}}{K_{\star}}\left(\ln (2)-\frac{7}{6}+2 \kappa_{2}^{[4]}\right)+\mathcal{O}\left(\frac{P^{4}}{K_{\star}^{2}}\right)\right\} .
$$

Consistency of (A.49) and (A.50) makes a prediction

$$
\kappa_{2}^{[4]}=\frac{13}{12}-\frac{1}{2} \ln (2) .
$$

\footnotetext{
${ }^{13}$ We used from (A.19) $\lambda_{2,2}^{h}=\left(-\frac{1}{4}+\frac{3}{4} \lambda_{2,0}^{h}\right)$.
} 
As a highly non-trivial check on our numerical analysis of the perturbative expansion, note that (A.51) agrees with (A.26) to within a factor of order $10^{-10}$.

With (A.51) we can also evaluate the speed of sound squared

$$
c_{s}^{2} \equiv \frac{\partial \mathcal{P}}{\partial \mathcal{E}}=\frac{1}{3}-\frac{4}{9} \frac{P^{2}}{K_{\star}}+\frac{10}{27} \frac{P^{4}}{K_{\star}^{2}}+\mathcal{O}\left(\frac{P^{6}}{K_{\star}^{3}}\right) .
$$

\section{A.3.3 First law of thermodynamics to order $\mathcal{O}\left(\frac{P^{6}}{K_{\star}^{3}}\right)$}

For the temperature dependence of $K_{\star}$ to order $\mathcal{O}\left(\frac{P^{6}}{K_{\star}^{3}}\right)$ we can again find two expressions - one involving both the UV parameters and the IR parameters, and the other one parameter-independent. These parallel the expressions (A.50) and (A.49) :

$$
\begin{gathered}
\frac{d K_{\star}}{d T}=\frac{2 P^{2}}{T}\left\{1+\frac{P^{2}}{K_{\star}}+\frac{P^{4}}{K_{\star}^{2}}\left(-\frac{1}{480} \pi^{4}-\frac{1}{30} \pi^{2}+\frac{1}{9} \ln (2)+\frac{71}{180}-\frac{2}{15}\left(\lambda_{2,0}^{h}\right)^{2}+\frac{8}{3} \kappa_{4,0}^{h}\right.\right. \\
\left.\left.+4 \xi_{4,0}^{h}-8 \xi_{4,2}^{h}+\zeta_{2}^{[4]}+2 \kappa_{2}^{[6]}\right)+\mathcal{O}\left(\frac{P^{6}}{K_{\star}^{3}}\right)\right\} \\
\frac{d K_{\star}}{d T}=\frac{2 P^{2}}{T}\left\{1+\frac{P^{2}}{K_{\star}}+\frac{1}{2} \frac{P^{4}}{K_{\star}^{2}}+\mathcal{O}\left(\frac{P^{6}}{K_{\star}^{3}}\right)\right\}
\end{gathered}
$$

where we used (A.34). Consistency of (A.53) and (A.54) leads to a prediction

$$
-\frac{1}{480} \pi^{4}-\frac{1}{30} \pi^{2}+\frac{1}{9} \ln (2)+\frac{71}{180}-\frac{2}{15}\left(\lambda_{2,0}^{h}\right)^{2}+\frac{8}{3} \kappa_{4,0}^{h}+4 \xi_{4,0}^{h}-8 \xi_{4,2}^{h}+\zeta_{2}^{[4]}+2 \kappa_{2}^{[6]}=\frac{1}{2} \text {. }
$$

We can estimate the error in our solutions by comparing the two sides of (A.55). Using the explicit expressions for the perturbative UV parameters (A.27) and the perturbative IR parameters (A.30) and (A.31), we find

$$
\left|\frac{L H S}{R H S}-1\right| \sim 2 \times 10^{-5} .
$$

Finally, the speed of sound can be expressed either in terms of the UV parameters or the IR parameters

$$
\begin{aligned}
c_{s}^{2}= & \frac{1}{3}-\frac{4}{9} \frac{P^{2}}{K_{\star}}+\frac{10}{27} \frac{P^{4}}{K_{\star}^{2}}+\left(-\frac{16}{81}-\frac{4}{9} \zeta_{2}^{[4]}-\frac{8}{9} \kappa_{2}^{[6]}\right) \frac{P^{6}}{K_{\star}^{3}}+\mathcal{O}\left(\frac{P^{8}}{K_{\star}^{4}}\right), \\
c_{s}^{2}= & \frac{1}{3}-\frac{4}{9} \frac{P^{2}}{K_{\star}}+\frac{10}{27} \frac{P^{4}}{K_{\star}^{2}}+\left(-\frac{1}{1080} \pi^{4}-\frac{2}{135} \pi^{2}+\frac{4}{81} \ln (2)-\frac{11}{45}-\frac{8}{135}\left(\lambda_{2,0}^{h}\right)^{2}\right. \\
& \left.+\frac{32}{27} \kappa_{4,0}^{h}+\frac{16}{9} \xi_{4,0}^{h}-\frac{32}{9} \xi_{4,2}^{h}\right) \frac{P^{6}}{K_{\star}^{3}}+\mathcal{O}\left(\frac{P^{8}}{K_{\star}^{4}}\right) .
\end{aligned}
$$

Consistency of (A.57) and (A.58) is guaranteed by (A.55). 


\section{References}

[1] J. M. Maldacena, "The large N limit of superconformal field theories and supergravity," Adv. Theor. Math. Phys. 2, 231 (1998) [Int. J. Theor. Phys. 38, 1113 (1999)] [arXiv:hep-th/9711200].

[2] S. S. Gubser, I. R. Klebanov and A. M. Polyakov, "Gauge theory correlators from non-critical string theory," Phys. Lett. B 428 (1998) 105 [arXiv:hep-th/9802109].

[3] E. Witten, "Anti-de Sitter space and holography," Adv. Theor. Math. Phys. 2, 253 (1998) [arXiv:hep-th/9802150].

[4] O. Aharony, S. S. Gubser, J. M. Maldacena, H. Ooguri and Y. Oz, "Large N field theories, string theory and gravity," Phys. Rept. 323, 183 (2000) [arXiv:hepth/9905111].

[5] E. Witten, "Anti-de Sitter space, thermal phase transition, and confinement in gauge theories," Adv. Theor. Math. Phys. 2, 505 (1998) [arXiv:hep-th/9803131].

[6] O. Aharony, J. Sonnenschein and S. Yankielowicz, "A holographic model of deconfinement and chiral symmetry restoration," Annals Phys. 322, 1420 (2007) [arXiv:hep-th/0604161].

[7] I. R. Klebanov and N. A. Nekrasov, "Gravity duals of fractional branes and logarithmic RG flow," Nucl. Phys. B 574, 263 (2000) [arXiv:hep-th/9911096].

[8] I. R. Klebanov and A. A. Tseytlin, "Gravity duals of supersymmetric SU(N) x SU(N+M) gauge theories," Nucl. Phys. B 578, 123 (2000) [arXiv:hep-th/0002159].

[9] I. R. Klebanov and M. J. Strassler, "Supergravity and a confining gauge theory: Duality cascades and chiSB-resolution of naked singularities," JHEP 0008, 052 (2000) [arXiv:hep-th/0007191].

[10] S. S. Gubser, C. P. Herzog, I. R. Klebanov and A. A. Tseytlin, "Restoration of chiral symmetry: A supergravity perspective," JHEP 0105, 028 (2001) [arXiv:hepth/0102172].

[11] A. Buchel, "Finite temperature resolution of the Klebanov-Tseytlin singularity," Nucl. Phys. B 600, 219 (2001) [arXiv:hep-th/0011146]. 
[12] M. Krasnitz, "A two point function in a cascading $\mathrm{N}=1$ gauge theory from supergravity," arXiv:hep-th/0011179.

[13] L. A. Pando Zayas and C. A. Terrero-Escalante, "Black holes with varying flux: A numerical approach," JHEP 0609, 051 (2006) [arXiv:hep-th/0605170].

[14] O. Aharony, "A note on the holographic interpretation of string theory backgrounds with varying flux," JHEP 0103, 012 (2001) [arXiv:hep-th/0101013].

[15] I. R. Klebanov, P. Ouyang and E. Witten, "A gravity dual of the chiral anomaly," Phys. Rev. D 65 (2002) 105007 [arXiv:hep-th/0202056].

[16] S. S. Gubser, C. P. Herzog and I. R. Klebanov, "Symmetry breaking and axionic strings in the warped deformed conifold," JHEP 0409, 036 (2004) [arXiv:hepth/0405282].

[17] O. Aharony and A. Buchel, in preparation.

[18] A. Buchel, "Transport properties of cascading gauge theories," Phys. Rev. D 72, 106002 (2005) [arXiv:hep-th/0509083].

[19] A. Buchel, "On jet quenching parameters in strongly coupled non-conformal gauge theories," Phys. Rev. D 74, 046006 (2006) [arXiv:hep-th/0605178].

[20] E. Caceres and A. Guijosa, "On drag forces and jet quenching in strongly coupled plasmas," JHEP 0612, 068 (2006) [arXiv:hep-th/0606134].

[21] T. Sakai and J. Sonnenschein, "Probing flavored mesons of confining gauge theories by supergravity," JHEP 0309, 047 (2003) [arXiv:hep-th/0305049].

[22] P. Ouyang, "Holomorphic D7-branes and flavored N = 1 gauge theories," Nucl. Phys. B 699 (2004) 207 [arXiv:hep-th/0311084].

[23] B. A. Burrington, J. T. Liu, L. A. Pando Zayas and D. Vaman, "Holographic duals of flavored $\mathrm{N}=1$ super Yang-Mills: Beyond the probe approximation," JHEP 0502 (2005) 022 [arXiv:hep-th/0406207].

[24] D. Arean, D. E. Crooks and A. V. Ramallo, "Supersymmetric probes on the conifold," JHEP 0411 (2004) 035 [arXiv:hep-th/0408210]. 
[25] S. Kuperstein, "Meson spectroscopy from holomorphic probes on the warped deformed conifold," JHEP 0503, 014 (2005) [arXiv:hep-th/0411097].

[26] T. S. Levi and P. Ouyang, "Mesons and Flavor on the Conifold," arXiv:hepth/0506021.

[27] F. Benini, F. Canoura, S. Cremonesi, C. Nunez and A. V. Ramallo, "Backreacting Flavors in the Klebanov-Strassler Background," arXiv:0706.1238 [hep-th].

[28] O. Aharony, talk at conference on "String Theory : Achievements and Perspectives", Tel Aviv, Israel, April 2007, http://stringfest.tau.ac.il/aharony.pdf

[29] M. J. Strassler, "The duality cascade," arXiv:hep-th/0505153.

[30] O. Aharony, A. Buchel and A. Yarom, "Holographic renormalization of cascading gauge theories," Phys. Rev. D 72, 066003 (2005) [arXiv:hep-th/0506002].

[31] O. Aharony, A. Buchel and A. Yarom, "Short distance properties of cascading gauge theories," JHEP 0611, 069 (2006) [arXiv:hep-th/0608209].

[32] A. Buchel, C. P. Herzog, I. R. Klebanov, L. A. Pando Zayas and A. A. Tseytlin, "Non-extremal gravity duals for fractional D3-branes on the conifold," JHEP 0104, 033 (2001) [arXiv:hep-th/0102105].

[33] I. R. Klebanov and E. Witten, "Superconformal field theory on threebranes at a Calabi-Yau singularity," Nucl. Phys. B 536, 199 (1998) [arXiv:hep-th/9807080].

[34] A. Buchel and J. T. Liu, "Thermodynamics of the $\mathrm{N}=2^{*}$ flow," JHEP 0311, 031 (2003) [arXiv:hep-th/0305064].

[35] C. P. Herzog, I. R. Klebanov and P. Ouyang, "Remarks on the warped deformed conifold," arXiv:hep-th/0108101.

[36] A. Buchel and J. T. Liu, "Universality of the shear viscosity in supergravity," Phys. Rev. Lett. 93, 090602 (2004) [arXiv:hep-th/0311175].

[37] A. Buchel, S. Deakin, P. Kerner and J. T. Liu, "Thermodynamics of the $\mathrm{N}=2^{*}$ strongly coupled plasma," arXiv:hep-th/0701142. 
[38] F. Karsch and E. Laermann, "Thermodynamics and in-medium hadron properties from lattice QCD," arXiv:hep-lat/0305025. 